\title{
Central Limit Theorems for Super Ornstein-Uhlenbeck Processes
}

\author{
Yan-Xia Ren · Renming Song • Rui Zhang
}

Received: 22 December 2012 / Accepted: 11 July 2013 / Published online: 8 August 2013

(C) Springer Science+Business Media Dordrecht 2013

\begin{abstract}
Suppose that $X=\left\{X_{t}: t \geq 0\right\}$ is a supercritical super Ornstein-Uhlenbeck process, that is, a superprocess with an Ornstein-Uhlenbeck process on $\mathbb{R}^{d}$ corresponding to $L=\frac{1}{2} \sigma^{2} \Delta-b x \cdot \nabla$ as its underlying spatial motion and with branching mechanism $\psi(\lambda)=-\alpha \lambda+\beta \lambda^{2}+\int_{(0,+\infty)}\left(e^{-\lambda x}-1+\lambda x\right) n(d x)$, where $\alpha=-\psi^{\prime}(0+)>0, \beta \geq 0$, and $n$ is a measure on $(0, \infty)$ such that $\int_{(0,+\infty)} x^{2} n(d x)<+\infty$. Let $\mathbb{P}_{\mu}$ be the law of $X$ with initial measure $\mu$. Then the process $W_{t}=e^{-\alpha t}\left\|X_{t}\right\|$ is a positive $\mathbb{P}_{\mu}$-martingale. Therefore there is $W_{\infty}$ such that $W_{t} \rightarrow W_{\infty}, \mathbb{P}_{\mu}$-a.s. as $t \rightarrow \infty$. In this paper we establish some spatial central limit theorems for $X$.
\end{abstract}

Let $\mathcal{P}$ denote the function class

$$
\mathcal{P}:=\left\{f \in C\left(\mathbb{R}^{d}\right): \text { there exists } k \in \mathbb{N} \text { such that }|f(x)| /\|x\|^{k} \rightarrow 0 \text { as }\|x\| \rightarrow \infty\right\}
$$

For each $f \in \mathcal{P}$ we define an integer $\gamma(f)$ in term of the spectral decomposition of $f$. In the small branching rate case $\alpha<2 \gamma(f) b$, we prove that there is constant $\sigma_{f}^{2} \in(0, \infty)$ such

Y.-X. Ren research was supported by NSFC (Grant No. 10871103 and 10971003) and Specialized Research Fund for the Doctoral Program of Higher Education.

R. Song research was supported in part by a grant from the Simons Foundation (208236).

R. Zhang was supported by the China Scholarship Council.

Y.-X. Ren

LMAM School of Mathematical Sciences \& Center for Statistical Science, Peking University, Beijing, 100871, P.R. China

e-mail: yxren@math.pku.edu.cn

R. Song

Department of Mathematics, University of Illinois, Urbana, IL 61801, USA

e-mail: rsong@math.uiuc.edu

R. Zhang $(\bowtie)$

LMAM School of Mathematical Sciences, Peking University, Beijing, 100871, P.R. China

e-mail: ruizhang8197@gmail.com 
that, conditioned on no-extinction,

$$
\left(e^{-\alpha t}\left\|X_{t}\right\|, \frac{\left\langle f, X_{t}\right\rangle}{\sqrt{\left\|X_{t}\right\|}}\right) \stackrel{d}{\rightarrow}\left(W^{*}, G_{1}(f)\right), \quad t \rightarrow \infty
$$

where $W^{*}$ has the same distribution as $W_{\infty}$ conditioned on no-extinction and $G_{1}(f) \sim$ $\mathcal{N}\left(0, \sigma_{f}^{2}\right)$. Moreover, $W^{*}$ and $G_{1}(f)$ are independent. In the critical rate case $\alpha=2 \gamma(f) b$, we prove that there is constant $\rho_{f}^{2} \in(0, \infty)$ such that, conditioned on no-extinction,

$$
\left(e^{-\alpha t}\left\|X_{t}\right\|, \frac{\left\langle f, X_{t}\right\rangle}{t^{1 / 2} \sqrt{\left\|X_{t}\right\|}}\right) \stackrel{d}{\rightarrow}\left(W^{*}, G_{2}(f)\right), \quad t \rightarrow \infty
$$

where $W^{*}$ has the same distribution as $W_{\infty}$ conditioned on no-extinction and $G_{2}(f) \sim$ $\mathcal{N}\left(0, \rho_{f}^{2}\right)$. Moreover $W^{*}$ and $G_{2}(f)$ are independent. We also establish two central limit theorems in the large branching rate case $\alpha>2 \gamma(f) b$.

Our central limit theorems in the small and critical branching rate cases sharpen the corresponding results in the recent preprint of Miłoś in that our limit normal random variables are non-degenerate. Our central limit theorems in the large branching rate case have no counterparts in the recent preprint of Miłoś. The main ideas for proving the central limit theorems are inspired by the arguments in K. Athreya's 3 papers on central limit theorems for continuous time multi-type branching processes published in the late 1960's and early 1970's.

Keywords Central limit theorem · Backbone decomposition · Superprocess $\cdot$ Super Ornstein-Uhlenbeck process · Branching process $\cdot$ Branching Ornstein-Uhlenbeck process $\cdot$ Ornstein-Uhlenbeck process

Mathematics Subject Classification (2000) Primary 60J80 · Secondary 60G57 · 60J45

\section{Introduction}

\subsection{Model}

Throughout this paper, $d \geq 1$ is an integer and $b>0$ is a number. We use $\xi=\left\{\xi_{t}: t \geq 0\right\}$ to denote an Ornstein-Uhlenbeck process (OU process, for short) on $\mathbb{R}^{d}$, that is, a diffusion process with infinitesimal generator

$$
L:=\frac{1}{2} \sigma^{2} \Delta-b x \cdot \nabla .
$$

For any $x \in \mathbb{R}^{d}$, we use $\Pi_{x}$ to denote the law of $\xi$ starting from $x$. The semigroup of $\xi$ will be denoted by $\left\{T_{t}: t \geq 0\right\}$.

Consider a branching mechanism of the form

$$
\psi(\lambda)=-\alpha \lambda+\beta \lambda^{2}+\int_{(0,+\infty)}\left(e^{-\lambda x}-1+\lambda x\right) n(d x), \quad \lambda>0,
$$

where $\alpha=-\psi^{\prime}(0+)>0, \beta \geq 0$, and $n$ is a measure on $(0, \infty)$ such that

$$
\int_{(0,+\infty)} x^{2} n(d x)<+\infty .
$$


Let $\mathcal{M}_{F}\left(\mathbb{R}^{d}\right)$ be the space of finite measures on $\mathbb{R}^{d}$. In this paper we will always assume that $X=\left\{X_{t}: t \geq 0\right\}$ is a super-Ornstein-Uhlenbeck process (super-OU process, for short) with underlying spatial motion $\xi$ and branching mechanism $\psi$. We will sometimes call $X$ a $(\xi, \psi)$-superprocess. The existence of such superprocesses is well-known, see, for instance, [13]. $X$ is a Markov branching process taking values in $\mathcal{M}_{F}\left(\mathbb{R}^{d}\right)$. For any $\mu \in \mathcal{M}_{F}\left(\mathbb{R}^{d}\right)$, we denote the law of $X$ with initial configuration $\mu$ by $\mathbb{P}_{\mu}$. The total mass of the process $X$ is a continuous-state branching process with branching mechanism $\psi$. The assumption (1.3) implies that the total mass process of $X$ does not explode. Since we always assume that $\alpha>0, X$ is a supercritical superprocess.

Let $\mathcal{B}_{b}^{+}\left(\mathbb{R}^{d}\right)$ be the space of positive, bounded measurable functions on $\mathbb{R}^{d}$. As usual, $\langle f, \mu\rangle:=\int f(x) \mu(d x)$ and $\|\mu\|:=\langle 1, \mu\rangle$. Then for every $f \in \mathcal{B}_{b}^{+}\left(\mathbb{R}^{d}\right)$ and $\mu \in \mathcal{M}_{F}\left(\mathbb{R}^{d}\right)$,

$$
-\log \mathbb{P}_{\mu}\left(e^{-\left\langle f, X_{t}\right\rangle}\right)=\left\langle u_{f}(\cdot, t), \mu\right\rangle,
$$

where $u_{f}(x, t)$ is the unique positive solution to the equation

$$
u_{f}(x, t)+\Pi_{x} \int_{0}^{t} \psi\left(u_{f}\left(\xi_{s}, t-s\right)\right) d s=\Pi_{x} f\left(\xi_{t}\right) .
$$

In addition, we assume that $\psi(\infty)=\infty$ which implies that the probability of the extinction event $\mathcal{E}:=\left\{\lim _{t \rightarrow \infty}\left\|X_{t}\right\|=0\right\}$ is contained in $(0,1)$, see for example the summary at the end of [23, Sect. 10.2.2]. Since $\psi$ is convex with $\psi(0)=0, \psi(\infty)=\infty$ and $\psi^{\prime}(0+)<0$, $\psi$ has exactly two roots in $[0, \infty)$; let $\lambda^{*}$ be the larger one. We have

$$
\mathbb{P}_{\mu}\left(\lim _{t \rightarrow \infty}\left\|X_{t}\right\|=0\right)=e^{-\lambda^{*}\|\mu\|}
$$

Using the expectation formula of $\left\|X_{t}\right\|$ and the Markov property of $X$, it is not hard to prove that (see Lemma 3.1 for a proof), under $\mathbb{P}_{\mu}$, the process $W_{t}=e^{-\alpha t}\left\|X_{t}\right\|$ is a positive martingale. Therefore it converges:

$$
W_{t} \rightarrow W_{\infty}, \quad \mathbb{P}_{\mu} \text {-a.s. } \quad \text { as } t \rightarrow \infty .
$$

Using the assumption (1.3) one can show that, as $t \rightarrow \infty, W_{t}$ also converges in $L^{2}\left(\mathbb{P}_{\mu}\right)$, so $W_{\infty}$ is non-degenerate and the second moment is finite. Moreover, we have $\mathbb{P}_{\mu}\left(W_{\infty}\right)=\|\mu\|$ and $\left\{W_{\infty}=0\right\}=\mathcal{E}$.

The purpose of this paper is to establish some spatial central limit theorems for the superOU process. More precisely, we want to find $A_{t}$ and $C_{t}$, for suitable test functions $f$, such that $C_{t}\left(\left\langle f, X_{t}\right\rangle-A_{t}\right)$ converges to some non-degenerate normal random variable as $t \rightarrow \infty$. It turns out that $C_{t}$ is determined by the second moment of $\left\langle f, X_{t}\right\rangle$ which depends on the sign of $\alpha-2 \gamma(f) b$, where $\gamma(f)$ is a quantity to be defined later.

There are many papers studying laws of large numbers for branching processes, branching diffusions and superprocesses. For example, see [2, 3, 17] for branching processes, [10, $11,31]$ for branching diffusions and [16, 18, 26, 27] for superprocesses. For super-OU processes with binary branching mechanism, the following weak law of large numbers was proved in [18]:

$$
e^{-\alpha t}\left\langle f, X_{t}\right\rangle \rightarrow\langle f, \varphi\rangle W_{\infty}, \quad \text { in probability }
$$

where $f \in C_{c}^{+}\left(\mathbb{R}^{d}\right), \varphi(x)=\left(\frac{b}{\pi \sigma^{2}}\right)^{d / 2} \exp \left(-\frac{b}{\sigma^{2}}\|x\|^{2}\right)$ and $\langle f, \varphi\rangle=\int_{\mathbb{R}^{d}} f(x) \varphi(x) d x$. When $\langle f, \varphi\rangle=0$, it is natural to consider central limit theorems for $\left\langle f, X_{t}\right\rangle$, that is, to find a normalization $C_{t}$ so that $C_{t}\left\langle f, X_{t}\right\rangle$ converges to a non-degenerate Gaussian random variable 
as $t \rightarrow \infty$. For branching processes, there are already papers dealing with central limit theorems. In 1966, Kesten and Stigum [21, 22] gave a central limit theorem for multi-type Galton-Watson processes by using the Jordan canonical form of the expectation matrix $M$. Then in [6-8], Athreya proved central limit theorems for multi-type continuous time branching processes; the main tools used in [6-8] are also the Jordan canonical form and the eigenvectors of the matrix $M_{t}$, the mean matrix at time $t$. Asmussen, Hering and Keiding discussed central limit theorems for some general branching Markov processes, see $[4,5]$ for example. Recently, central limit theorems for branching OU particle systems and for super-OU processes were established in [1] and [29] respectively. However, the limiting normal random variables in the central limit theorems in [1,29] (see [1, Theorems 3.2 and 3.6] and [29, Theorems 3 and 4]) may be degenerate (i.e., equal to zero), so the central limit theorems in $[1,29]$ are not completely satisfactory.

In this paper, we sharpen the results of [29] and establish central limit theorems for superOU processes which are more satisfactory in the sense that the limiting normal random variables in our results are non-degenerate. The setup of this paper is more general than that of [29] since we allow a general branching mechanism as opposed to the binary branching mechanism in [29]. The only assumption on the branching mechanism is the second moment condition (1.3), which is necessary for central limit theorems.

We mention that we are following Athreya's argument for multi-type (finite type) branching processes, also called multidimensional Galton-Watson processes, and show that Athreya's ideas for multi-type branching processes also work for super-OU processes, which can be regarded as an infinite-type branching process. The main tool of this paper is, similar to that of [29], also the backbone decomposition of supercritical superprocesses, see [9]. The main idea of the backbone decomposition is that a supercritical super-OU process can be constructed from a branching OU process (known as the backbone), in which particles live forever (known as immortal particles). After dressing the backbone with subcritical super-OU processes, we get a measure-valued Markov process, which gives a version of the super-OU process. Since subcritical super-OU process will become extinct in finite time, we can imagine that the limit behavior of super-OU process is determined by the backbone branching OU process. In this paper we prove that these intuitive ideas work well. For the precise backbone decomposition, see Sect. 2.1.

We remark here that, under the extra condition $\int^{\infty} \frac{1}{\psi(\lambda)} d \lambda<\infty$ on the branching mechanism, we could use the excursion measures $\left\{\mathbb{N}_{x}, x \in \mathbb{R}^{d}\right\}$ of our superprocess instead of the backbone decomposition to prove our central limit theorems. In fact, in this case, the arguments are somewhat shorter. We choose to use the backbone decomposition argument since we could not make the excursion measure argument work in the general case. The main difficulty is that $\mathbb{N}_{x}$ is not a finite measure. Under the assumption $\int^{\infty} \frac{1}{\psi(\lambda)} d \lambda<\infty$, the event $\mathcal{E}$ is equal to the event $\left\{\zeta_{0}<\infty\right\}, \mathbb{P}_{\mu}$-a.s., where $\zeta_{0}=\inf \left\{t>0,\left\|X_{t}\right\|=0\right\}$, and more importantly, $\mathbb{N}_{x}\left(\cdot, \zeta_{0}>t\right)<\infty$ is a finite and non-zero measure for any $t>0$. Without the extra assumption, it might happen that $\mathbb{P}_{\delta_{x}}\left(\zeta_{0} \leq t\right)=0$ for all $t>0$ and thus $\mathbb{N}_{x}\left(\zeta_{0}>t\right)=\infty$.

The main feature of the super OU processes used in this paper is that the spectrum of the generator of the OU process is discrete and that eigenfunctions form a complete orthonormal basis in a certain $L^{2}$ space. A natural and important follow-up question is the following: can one extend the central limit theorems to supercritical superprocesses with general spatial motion and with spatial-dependent branching mechanism? In the sequel [30] to the present paper, we formulated a general setup and established spatial central limit theorems for supercritical branching Markov processes with general spatial motion and general spatial-dependent branching mechanism. By combining the ideas of the present paper with 
that of [30], one could extend the central limit theorems of this paper to supercritical superprocesses with general spatial motion and with spatial-dependent branching mechanism. We will carry this out later.

\subsection{Eigenfunctions of OU Processes}

Recall that $\left\{T_{t}, t \geq 0\right\}$ is the semigroup of the OU process $\xi$. It is well-known that $\xi$ has an invariant density

$$
\varphi(x)=\left(\frac{b}{\pi \sigma^{2}}\right)^{d / 2} \exp \left(-\frac{b}{\sigma^{2}}\|x\|^{2}\right) .
$$

Let $L^{2}(\varphi):=\left\{h: \int_{\mathbb{R}^{d}}|h(x)|^{2} \varphi(x) d x<\infty\right\}$. For $h_{1}, h_{2} \in L^{2}(\varphi)$, we define

$$
\left\langle h_{1}, h_{2}\right\rangle_{\varphi}:=\int_{\mathbb{R}^{d}} h_{1}(x) h_{2}(x) \varphi(x) d x .
$$

In this subsection, we recall some results on the spectrum in $L^{2}(\varphi)$ of the operator $L$ defined in (1.1), more details can be found in [28]. For $p=\left(p_{1}, p_{2}, \ldots, p_{d}\right) \in \mathbb{Z}_{+}^{d}$, let $|p|=\sum_{j=1}^{d} p_{j}$ and $p !=\prod_{j=1}^{d} p_{j}$ !. Recall the Hermite polynomials $\left\{H_{p}(x), p \in \mathbb{Z}_{+}^{d}\right\}$ :

$$
H_{p}(x)=(-1)^{|p|} e^{\|x\|^{2}} \frac{\partial}{\partial x_{1}^{p_{1}} \cdots \partial x_{d}^{p_{d}}}\left(e^{-\|x\|^{2}}\right) .
$$

The eigenvalues of $L$ are $\{-m b, m=0,1,2, \ldots\}$ and the corresponding eigenspaces $A_{m}$ are given by

$$
A_{m}:=\operatorname{Span}\left\{\phi_{p},|p|=m\right\},
$$

where

$$
\phi_{p}(x)=\frac{1}{\sqrt{p ! 2^{|p|}}} H_{p}\left(\frac{\sqrt{b}}{\sigma} x\right) .
$$

In particular, $\phi_{0,0, \ldots, 0}(x)=1, \phi_{e_{i}}(x)=\frac{\sqrt{2 b}}{\sigma} x_{i}$, where $e_{i}$ stands for the unit vector in the $x_{i}$ direction. The function $\phi_{p}$ is an eigenfunction of $L$ corresponding to the eigenvalue $-|p| b$ and therefore

$$
T_{t} \phi_{p}(x)=e^{-|p| b t} \phi_{p}(x) .
$$

Moreover, the eigenfunctions $\left\{\phi_{p}(x), p \in \mathbb{Z}_{+}^{d}\right\}$ form a complete orthonormal basis for $L^{2}(\varphi)$. Thus every $f \in L^{2}(\varphi)$ admits the following $L^{2}(\varphi)$ expansion:

$$
f(x)=\sum_{m=0}^{\infty} \sum_{|p|=m} a_{p} \phi_{p}(x),
$$

where $a_{p}=\left\langle f, \phi_{p}\right\rangle_{\varphi}$. Define

$$
\gamma(f):=\inf \left\{n \geq 0: \text { there exists } p \in \mathbb{Z}_{+}^{d} \text { with }|p|=n \text { such that } a_{p} \neq 0\right\},
$$

where we use the usual convention $\inf \varnothing=\infty$. In this paper we will use $\mathcal{P}$ to denote the function class

$$
\mathcal{P}:=\left\{f \in C\left(\mathbb{R}^{d}\right): \text { there exists } k \in \mathbb{N} \text { such that }|f(x)| /\|x\|^{k} \rightarrow 0 \text { as }\|x\| \rightarrow \infty\right\} .
$$


We easily see that $\mathcal{P} \subset L^{2}(\varphi)$ and for $f \in \mathcal{P}$, there exists $k \in \mathbb{N}$ such that

$$
|f(x)| \lesssim 1+\|x\|^{k}
$$

where we used the following notation: for two positive functions $f$ and $g, f(x) \lesssim g(x)$ means that there exists a constant $c>0$ such that $f(x) \leq c g(x)$.

\subsection{Main Results for Super-OU Processses}

In this subsection we give the main results of this paper. The proofs will be given in the later sections. In the remainder of this paper, whenever we deal with an initial configuration $\mu \in \mathcal{M}_{F}\left(\mathbb{R}^{d}\right)$, we are implicitly assuming that it has compact support.

\subsubsection{Large Branching Rate: $\alpha>2 b \gamma(f)$}

For each $p \in \mathbb{Z}_{+}^{d}$, we define

$$
H_{t}^{p}:=e^{-(\alpha-|p| b) t}\left\langle\phi_{p}, X_{t}\right\rangle, \quad t \geq 0
$$

Then one can show (see Lemma 3.1 below) that, if $\alpha>2|p| b, H_{t}^{p}$ is a $\mathbb{P}_{\mu}$-martingale bounded in $L^{2}\left(\mathbb{P}_{\mu}\right)$, and thus the limit $H_{\infty}^{p}:=\lim _{t \rightarrow \infty} H_{t}^{p}$ exists $\mathbb{P}_{\mu}$-a.s. and in $L^{2}\left(\mathbb{P}_{\mu}\right)$.

Theorem 1.1 If $f \in \mathcal{P}$ satisfies $\alpha>2 \gamma(f) b$, then as $t \rightarrow \infty$,

$$
e^{-(\alpha-\gamma(f) b) t}\left\langle f, X_{t}\right\rangle \rightarrow \sum_{|p|=\gamma(f)} a_{p} H_{\infty}^{p}, \quad \text { in } L^{2}\left(\mathbb{P}_{\mu}\right)
$$

Remark 1.2 When $\gamma(f)=0, H_{t}^{0}$ reduces to $W_{t}$, and thus $H_{\infty}^{0}=W_{\infty}$. Therefore by Theorem 1.1 and the fact that $a_{0}=\langle f, \varphi\rangle$, we get that, as $t \rightarrow \infty$,

$$
e^{-\alpha t}\left\langle f, X_{t}\right\rangle \rightarrow\langle f, \varphi\rangle W_{\infty}, \quad \text { in } L^{2}\left(\mathbb{P}_{\mu}\right)
$$

In particular, the convergence also holds in $\mathbb{P}_{\mu}$-probability, so it implies the results in [18] in the case of super-OU processes. Moreover, by (1.6), on $\mathcal{E}^{c}$, we have

$$
\left\|X_{t}\right\|^{-1}\left\langle f, X_{t}\right\rangle \rightarrow\langle f, \varphi\rangle, \quad \text { in } \mathbb{P}_{\mu} \text {-probability. }
$$

Remark 1.3 We think that the convergence in Theorem 1.1 is also valid in the almost sure sense. In fact, from the proof below we see that the first-order term is a $L^{2}\left(\mathbb{P}_{\mu}\right)$-bounded martingale, and the second moment of the remainder term decays exponentially fast, which implies that we have almost sure convergence along the discrete time sequences $\{n \delta, n \geq 1\}$ for any $\delta>0$. However, we have not been able yet to prove the almost sure convergence for continuous time. In [10], Conner gave a proof of a similar almost sure convergence result for branching Brownian motion in a finite interval with absorbing boundary, however, his technology does not work in the present case. 


\subsubsection{Small Branching Rate: $\alpha<2 \gamma(f) b$}

Let

$$
\sigma_{f}^{2}:=A \int_{0}^{\infty} e^{\alpha s}\left\langle\left(T_{s} f\right)^{2}, \varphi\right\rangle d s=A \sum_{n=\gamma(f)}^{\infty} \sum_{|p|=n} \frac{a_{p}^{2}}{2 n b-\alpha}
$$

where

$$
A:=\psi^{(2)}(0+)=2 \beta+\int_{(0, \infty)} x^{2} n(d x)<\infty .
$$

Obviously, under the assumptions of this paper, $A \in(0, \infty)$. In the rest of this paper, $A$ will always stand for this constant.

Theorem 1.4 If $f \in \mathcal{P}$ satisfies $\alpha<2 \gamma(f) b$, then $\sigma_{f}^{2} \in(0, \infty)$ and, under $\mathbb{P}_{\mu}\left(\cdot \mid \mathcal{E}^{c}\right)$, it holds that

$$
\left(e^{-\alpha t}\left\|X_{t}\right\|, \frac{\left\langle f, X_{t}\right\rangle}{\sqrt{\left\|X_{t}\right\|}}\right) \stackrel{d}{\rightarrow}\left(W^{*}, G_{1}(f)\right), \quad t \rightarrow \infty
$$

where $W^{*}$ has the same distribution as $W_{\infty}$ conditioned on $\mathcal{E}^{c}$ and $G_{1}(f) \sim \mathcal{N}\left(0, \sigma_{f}^{2}\right)$. Moreover, $W^{*}$ and $G_{1}(f)$ are independent.

Remark 1.5 Using the theorem above, we get that if $\alpha<2 \gamma(f) b$, then, under $\mathbb{P}_{\mu}$, we have

$$
e^{-\alpha t / 2}\left\langle f, X_{t}\right\rangle \stackrel{d}{\rightarrow} G_{1}(f) \sqrt{W_{\infty}}
$$

where $G_{1}(f)$ is the same as in the theorem above.

\subsubsection{The Critical Case: $\alpha=2 \gamma(f) b$}

Define

$$
\rho_{f}^{2}:=A \sum_{|p|=\gamma(f)}\left(a_{p}\right)^{2}
$$

Obviously, $\rho_{f}^{2} \in(0, \infty)$.

Theorem 1.6 If $f \in \mathcal{P}$ satisfies $\alpha=2 \gamma(f) b$, then, under $\mathbb{P}_{\mu}\left(\cdot \mid \mathcal{E}^{c}\right)$, it holds that

$$
\left(e^{-\alpha t}\left\|X_{t}\right\|, \frac{\left\langle f, X_{t}\right\rangle}{t^{1 / 2} \sqrt{\left\|X_{t}\right\|}}\right) \stackrel{d}{\rightarrow}\left(W^{*}, G_{2}(f)\right), \quad t \rightarrow \infty
$$

where $W^{*}$ has the same distribution as $W_{\infty}$ conditioned on $\mathcal{E}^{c}, G_{2}(f) \sim \mathcal{N}\left(0, \rho_{f}^{2}\right)$. Moreover $W^{*}$ and $G_{2}(f)$ are independent.

Remark 1.7 Using the theorem above, we get that if $\alpha=2 \gamma(f) b$, then, under $\mathbb{P}_{\mu}$, we have

$$
t^{-1 / 2} e^{-\alpha t / 2}\left\langle f, X_{t}\right\rangle \stackrel{d}{\rightarrow} G_{2}(f) \sqrt{W_{\infty}}, \quad t \rightarrow \infty
$$

where $G_{2}(f)$ is the same as in the theorem above. 
Remark 1.8 Note that the limiting normal random variables in our Theorems 1.4 and 1.6 are non-degenerate.

Remark 1.9 The results of [29] correspond to the case $\gamma(f)=1$ in the present paper. For the small branching rate case of [29], the definition of $\sigma_{f}^{2}$ in [29, (3.1)] can be simplified and there is a minor mistake in [29, (3.1)]. In fact, using the notation of [29],

$$
\sigma_{f}^{2}=2 \beta \int_{0}^{\infty} e^{-\alpha s}\left\langle\varphi,\left(\mathcal{P}_{s}^{\alpha} \tilde{f}(\cdot)\right)^{2}\right\rangle d s
$$

$\tilde{f}(x)=f(x)-\langle f, \phi\rangle$, since it is easy to check that the sum of the last two parts of [29, (3.1)] is 0 , that is

$$
\int_{0}^{\infty}\left\langle\varphi,\left(-2 \beta\left(\mathcal{P}_{s}^{-\alpha} \tilde{f}(\cdot)\right)^{2}+4 \alpha \beta u(\cdot, s)\right)\right\rangle d s=0,
$$

where $u(x, s)=\int_{0}^{s} \mathcal{P}_{s-u}^{-\alpha}\left[\left(\mathcal{P}_{u}^{-\alpha} \tilde{f}(\cdot)\right)^{2}\right](x) d u$. Furthermore, the factor $\beta / \alpha$ on the right side of [29, (3.1)] should also be deleted. In the critical branching case of [29], the factor $\beta / \alpha$ on the right side of $[29,(3.2)]$ should be deleted. The correct form of $[29,(3.2)]$ should be (in the notation of [29])

$$
\sigma_{f}^{2}=2 \beta \int_{\mathbb{R}^{d}}(x \circ\langle\operatorname{grad}(f), \varphi\rangle)^{2} \varphi(x) d x .
$$

With these minor corrections, the results of [29] coincide with our Theorems 1.1, 1.4 and 1.6 when $\gamma(f)=1$.

For convenience, we introduce the following notation. For any $f \in L^{2}(\varphi)$, we define

$$
f_{(s)}(x)=\sum_{\gamma(f) \leq m<\alpha /(2 b)} \sum_{|p|=m} a_{p} \phi_{p}(x), \quad f_{(c)}(x)=\sum_{m=\alpha /(2 b)} \sum_{|p|=m} a_{p} \phi_{p}(x),
$$

and

$$
f_{(l)}(x)=f(x)-f_{(s)}(x)-f_{(c)}(x)=\sum_{m>\alpha /(2 b)}^{\infty} \sum_{|p|=m} a_{p} \phi_{p}(x) .
$$

Strictly speaking, $f_{(s)}, f_{(c)}, f_{(l)}$ also depend on $\alpha /(2 b)$. For simplicity of notation, we omit this dependence in the notation above.

Combining Theorems 1.1, 1.4 and 1.6, we have the following expansion of $\left\langle f, X_{t}\right\rangle$ : for any $f \in \mathcal{P}$,

$$
\begin{aligned}
\left\langle f, X_{t}\right\rangle= & \sum_{\gamma(f) \leq m<\frac{\alpha}{2 b}} \sum_{|p|=m} a_{p} e^{-(\alpha-m b) t}\left\langle\phi_{p}, X_{t}\right\rangle \cdot e^{(\alpha-m b) t} \\
& +\sum_{|p|=\frac{\alpha}{2 b}} a_{p} t^{-1 / 2} e^{-(\alpha / 2) t}\left\langle\phi_{p}, X_{t}\right\rangle \cdot \sqrt{t} e^{(\alpha / 2) t}+\left\langle f_{(l)}, X_{t}\right\rangle \\
= & \sum_{\gamma(f) \leq m<\frac{\alpha}{2 b}|p|=m} \sum_{p} a_{p} U_{p}(t) \cdot e^{(\alpha-m b) t}+\sum_{|p|=\frac{\alpha}{2 b}} a_{p} U_{p}(t) \cdot \sqrt{t} e^{\alpha t / 2}+\left\langle f_{(l)}, X_{t}\right\rangle,
\end{aligned}
$$


where

$$
U_{p}(t)= \begin{cases}e^{-(\alpha-|p| b) t}\left\langle\phi_{p}, X_{t}\right\rangle, & |p|<\frac{\alpha}{2 b}, \\ t^{-1 / 2} e^{-\alpha t / 2}\left\langle\phi_{p}, X_{t}\right\rangle, & |p|=\frac{\alpha}{2 b}\end{cases}
$$

Further, if $|p|<\frac{\alpha}{2 b}$, then $U_{p}(t)=H_{t}^{p}$ converges to $H_{\infty}^{p}, \mathbb{P}_{\mu}$-a.s. and in $L^{2}\left(\mathbb{P}_{\mu}\right)$; if $|p|=\frac{\alpha}{2 b}$, $U_{p}(t)$ converges in law to $G_{2}\left(\phi_{p}\right) \sqrt{W_{\infty}}$ with $G_{2}\left(\phi_{p}\right) \sim \mathcal{N}(0, A) ; e^{-(\alpha / 2) t}\left\langle f_{(l)}, X_{t}\right\rangle$ converges in law to $G_{1}\left(f_{(l)}\right) \sqrt{W_{\infty}}$.

\subsubsection{Further Results in the Large Branching Rate Case}

In this subsection we give two central limit theorems for the case $\alpha>2 \gamma(f) b$. These two theorems have no counterparts in [29]. Define

$$
H_{\infty}:=\sum_{\gamma(f) \leq m<\alpha /(2 b)} \sum_{|p|=m} a_{p} H_{\infty}^{p} .
$$

Let

$$
\beta_{f_{(s)}}^{2}:=A \sum_{\gamma(f) \leq m<\alpha /(2 b)} \frac{1}{\alpha-2 m b} \sum_{|p|=m} a_{p}^{2} .
$$

In Sect. 3.3 we will see that $\beta_{f_{(s)}}^{2}=\left\langle\mathbf{V} a r_{\delta_{x}} H_{\infty}, \varphi\right\rangle$.

Theorem 1.10 If $f \in \mathcal{P}$ satisfies $\alpha>2 \gamma(f) b$ and $f_{(c)}=0$, then $\beta_{f_{(s)}}^{2} \in(0, \infty)$. Under $\mathbb{P}_{\mu}\left(\cdot \mid \mathcal{E}^{c}\right)$, it holds that, as $t \rightarrow \infty$,

$$
\left(e^{-\alpha t}\left\|X_{t}\right\|,\left\|X_{t}\right\|^{-1 / 2}\left(\left\langle f, X_{t}\right\rangle-\sum_{\gamma(f)=m<\alpha /(2 b)} e^{(\alpha-m b) t} \sum_{|p|=m} a_{p} H_{\infty}^{p}\right)\right) \stackrel{d}{\rightarrow}\left(W^{*}, G_{3}(f)\right),
$$

where $W^{*}$ has the same distribution as $W_{\infty}$ conditioned on $\mathcal{E}^{c}$, and $G_{3}(f) \sim \mathcal{N}\left(0, \sigma_{f_{(l)}}^{2}+\right.$ $\left.\beta_{f_{(s)}}^{2}\right)$. Moreover, $W^{*}$ and $G_{3}(f)$ are independent.

Remark 1.11 If $\alpha>2|p| b$, then under $\mathbb{P}_{\mu}\left(\cdot \mid \mathcal{E}^{c}\right)$, it holds that, as $t \rightarrow \infty$,

$$
\left(e^{-\alpha t}\left\|X_{t}\right\|, \frac{\left(\left\langle\phi_{p}, X_{t}\right\rangle-e^{(\alpha-|p| b) t} H_{\infty}^{p}\right)}{\left\|X_{t}\right\|^{1 / 2}}\right) \stackrel{d}{\rightarrow}\left(W^{*}, G_{3}\right)
$$

where $G_{3} \sim \mathcal{N}\left(0, \frac{A}{\alpha-2|p| b}\right)$. In particular, for $|p|=0$, we have

$$
\left(e^{-\alpha t}\left\|X_{t}\right\|, \frac{\left\|X_{t}\right\|-e^{\alpha t} W_{\infty}}{\sqrt{\left\|X_{t}\right\|}}\right) \stackrel{d}{\rightarrow}\left(W^{*}, G_{3}\right), \quad t \rightarrow \infty
$$

where $G_{3} \sim \mathcal{N}\left(0, \frac{A}{\alpha}\right)$.

Remark 1.12 Using the theorem above, we get that if $\alpha>2 \gamma(f) b$ and $f_{(c)}=0$, then under $\mathbb{P}_{\mu}$, we have, as $t \rightarrow \infty$,

$$
\left(e^{-\alpha t}\left\|X_{t}\right\|, e^{-(\alpha / 2) t}\left(\left\langle f, X_{t}\right\rangle-\sum_{\gamma(f) \leq m<\alpha /(2 b)} e^{(\alpha-m b) t} \sum_{|p|=m} a_{p} H_{\infty}^{p}\right)\right) \stackrel{d}{\rightarrow}\left(W_{\infty}, \sqrt{W_{\infty}} G_{3}(f)\right),
$$

where $G_{3}(f)$ is the same as in the theorem above. 
Theorem 1.13 If $f \in \mathcal{P}$ satisfies $f_{(c)} \neq 0$, then, under $\mathbb{P}_{\mu}\left(\cdot \mid \mathcal{E}^{c}\right)$, it holds that, as $t \rightarrow \infty$,

$$
\left(e^{-\alpha t}\left\|X_{t}\right\|, t^{-1 / 2}\left\|X_{t}\right\|^{-1 / 2}\left(\left\langle f, X_{t}\right\rangle-\sum_{\gamma(f) \leq m<\alpha /(2 b)} e^{(\alpha-m b) t} \sum_{|p|=m} a_{p} H_{\infty}^{p}\right)\right) \stackrel{d}{\rightarrow}\left(W^{*}, G_{4}(f)\right),
$$

where $W^{*}$ has the same distribution as $W_{\infty}$ conditioned on $\mathcal{E}^{c}$, and

$$
G_{4}(f) \sim \mathcal{N}\left(0, A \sum_{|p|=\alpha / 2 b}\left(a_{p}\right)^{2}\right) .
$$

Moreover, $W^{*}$ and $G_{4}(f)$ are independent.

Remark 1.14 Note that the limiting normal random variables in our Theorems 1.10 and 1.13 are non-degenerate.

\section{Preliminaries}

\subsection{Backbone Decomposition of Super-OU Processes}

In this subsection, we recall the backbone decomposition of [9]. Define another branching mechanism $\psi^{*}$ by

$$
\begin{aligned}
\psi^{*}(\lambda) & =\psi\left(\lambda+\lambda^{*}\right) \\
& =\alpha^{*} \lambda+\beta \lambda^{2}+\int_{(0, \infty)}\left(e^{-\lambda x}-1+\lambda x\right) e^{-\lambda^{*} x} n(d x),
\end{aligned}
$$

where

$$
\alpha^{*}=-\alpha+2 \beta \lambda^{*}+\int_{(0, \infty)}\left(1-e^{-\lambda^{*} x}\right) x n(d x) .
$$

It is easy to see that $\alpha^{*}=\left(\psi^{*}\right)^{\prime}(0+)=\psi^{\prime}\left(\lambda^{*}\right)>0$. So the $\left(\xi, \psi^{*}\right)$-superprocess is subcritical. Note that it follows from (2.1) that the measure $n^{*}$ associated with $\psi^{*}$ is $e^{-\lambda^{*} x} n(d x)$, thus for any $n \in \mathbb{N}, \int_{0}^{\infty} x^{n} n^{*}(d x)<\infty$. It follows from [9, Lemma 2] that the $(\xi, \psi)$ superprocess conditioned on $\mathcal{E}$ has the same law as the $\left(\xi, \psi^{*}\right)$-superprocess. Let $\mathbb{P}_{\mu}^{*}$ be the law of the $\left(\xi, \psi^{*}\right)$-superprocess with initial configuration $\mu$, and define

$$
u_{f}^{*}(x, t)=-\log \mathbb{P}_{\delta_{x}}^{*}\left(e^{-\left\langle f, X_{t}\right\rangle}\right) .
$$

It was shown in [14] that one can associate with $\left\{\mathbb{P}_{\delta_{x}}^{*}: x \in \mathbb{R}^{d}\right\}$ a family of measures $\left\{\mathbb{N}_{x}^{*}: x \in \mathbb{R}^{d}\right\}$, defined on the same measurable space as the probabilities $\left\{\mathbb{P}_{\delta_{x}^{*}}^{*}: x \in \mathbb{R}^{d}\right\}$ and satisfying

$$
\mathbb{N}_{x}^{*}\left(1-e^{-\left\langle f, X_{t}\right\rangle}\right)=-\log \mathbb{P}_{\delta_{x}}^{*}\left(e^{-\left\langle f, X_{t}\right\rangle}\right)=u_{f}^{*}(x, t),
$$

for all $f \in \mathcal{B}_{b}^{+}\left(\mathbb{R}^{d}\right)$ and $t>0$. The branching property implies that $\mathbb{P}_{\delta_{x}}^{*}$ is an infinitely divisible measure on the path space of $X$, that is to say, the space of measure-valued cadlag functions, $\mathbb{D}\left([0, \infty), \mathcal{M}_{F}\left(\mathbb{R}^{d}\right)\right)$, and (2.2) is a Lévy-Khinchine formula in which $\mathbb{N}_{x}^{*}$ plays the role of its Lévy measure. The measures $\left\{\mathbb{N}_{x}^{*}: x \in \mathbb{R}^{d}\right\}$ are also called excursion measures, see[14] and [25] for further details on $\mathbb{N}_{x}^{*}$. For earlier work on excursion measures of superprocesses, see [15] and [24]. 
Let $\mathcal{M}_{a}\left(\mathbb{R}^{d}\right)$ be the space of finite integer-valued atomic measures on $\mathbb{R}^{d}$. For $v \in$ $\mathcal{M}_{a}\left(\mathbb{R}^{d}\right)$, let $Z=\left(Z_{t}: t \geq 0\right)$ be a branching OU-process with initial configuration $v$. $\left\{Z_{t}, t \geq 0\right\}$ is an $\mathcal{M}_{a}\left(\mathbb{R}^{d}\right)$-valued Markov process in which individuals, from the moment of birth, live for an independent and exponentially distributed period of time with parameter $\alpha^{*}$ during which they move according to the OU-process issued from their position of birth and at death they give birth at the same position to an independent number of offspring with distribution $\left(p_{n}: n \geq 0\right)$, where $p_{0}=p_{1}=0$ and for $n \geq 2$,

$$
p_{n}=\frac{1}{\lambda^{*} \alpha^{*}}\left\{\beta\left(\lambda^{*}\right)^{2} \mathbf{1}_{\{n=2\}}+\left(\lambda^{*}\right)^{n} \int_{(0, \infty)} \frac{x^{n}}{n !} e^{-\lambda^{*} x} n(d x)\right\} .
$$

The generator of $Z$ is given by

$$
F(s)=\alpha^{*} \sum_{n \geq 0} p_{n}\left(s^{n}-s\right)=\frac{1}{\lambda^{*}} \psi\left(\lambda^{*}(1-s)\right) .
$$

$Z$ is referred as the $(\xi, F)$-backbone in [9]. Moreover, when referring to individuals in $Z$ we will use the classical Ulam-Harris notation so that every particle in $Z$ has a unique label, see [20]. Let $\mathcal{T}$ be the set of labels of individuals realized in $Z$. Let $\left|Z_{t}\right|$ be the number of particles alive at time $t$. For each individual $u \in \mathcal{T}$ we shall write $\tau_{u}$ and $\sigma_{u}$ for its birth and death times respectively and $\left\{z_{u}(r): r \in\left[\tau_{u}, \sigma_{u}\right]\right\}$ for its spatial trajectory. Now we follow [9] and describe three kinds of immigrations along the backbone $Z$ as follows.

1. Continuous immigration: The process $I^{\mathbb{N}^{*}}$ is measure-valued on $\mathbb{R}^{d}$ such that

$$
I_{t}^{\mathbb{N}^{*}}:=\sum_{u \in \mathcal{T}} \sum_{u \wedge \tau_{u}<r \leq t \wedge \sigma_{u}} X_{t-r}^{(1, u, r)}
$$

where, given $Z$, independently for each $u \in \mathcal{T}$ with $\tau_{u}<t$, the processes $X^{(1, u, r)}$ are countable in number and correspond to $\mathbb{D}\left([0, \infty), \mathcal{M}_{F}\left(\mathbb{R}^{d}\right)\right)$-valued, Poissonian immigration along the time-space trajectory $\left\{\left(r, z_{u}(r)\right): r \in\left(\tau_{u}, t \wedge \sigma_{u}\right]\right\}$ with rate $2 \beta \mathrm{d} r \times$ $\mathrm{dN}_{z_{u}(r)}^{*}$.

2. Discontinuous immigration: The processes $I^{\mathbb{P}^{*}}$ is measure-valued on $\mathbb{R}^{d}$ such that

$$
I_{t}^{\mathbb{P}^{*}}:=\sum_{u \in \mathcal{T}} \sum_{t \wedge \tau_{u}<r \leq t \wedge \sigma_{u}} X_{t-r}^{(2, u, r)},
$$

where, given $Z$, independently for each $u \in \mathcal{T}$ with $\tau_{u}<t$, the processes $X^{(2, u, r)}$ are countable in number and correspond to $\mathbb{D}\left([0, \infty), \mathcal{M}_{F}\left(\mathbb{R}^{d}\right)\right)$-valued, Poissonian immigration along the time-space trajectory $\left\{\left(r, z_{u}(r)\right): r \in\left(\tau_{u}, t \wedge \sigma_{u}\right]\right\}$ with rate $\mathrm{d} r \times \int_{y \in(0, \infty)} y e^{-\lambda^{*} y} n(d y) \mathrm{d} \mathbb{P}_{y \delta_{z u}^{*}(r)}$.

3. Branching point biased immigration: The process $I^{\eta}$ is measure-valued on $\mathbb{R}^{d}$ such that

$$
I_{t}^{\eta}=\sum_{u \in \mathcal{T}} \mathbf{1}_{\sigma_{u} \leq t} X_{t-\sigma_{u}}^{(3, u)}
$$

where, given $Z$, independently for each $u \in \mathcal{T}$ with $\sigma_{u} \leq t$, the process $X^{(3, u)}$ is an independent copy of the canonical process $X$ issued at time $\sigma_{u}$ with law $\mathbb{P}_{Y_{u} \delta_{z_{u}\left(\sigma_{u}\right)}}$ where, given $u$ has $n \geq 2$ offspring, $Y_{u}$ is an independent random variable with distribution 
$\eta_{n}(\mathrm{~d} y)$, where

$$
\eta_{n}(d y)=\frac{1}{p_{n} \lambda^{*} \alpha^{*}}\left\{\beta\left(\lambda^{*}\right)^{2} \delta_{0}(d y) \mathbf{1}_{\{n=2\}}+\left(\lambda^{*}\right)^{n} \frac{y^{n}}{n !} e^{-\lambda^{*} y} n(d y)\right\} .
$$

Now we define another $\mathcal{M}_{F}\left(\mathbb{R}^{d}\right)$-valued process $I=\left\{I_{t}: t \geq 0\right\}$ by

$$
I:=I^{\mathbb{N}^{*}}+I^{\mathbb{P}^{*}}+I^{\eta}
$$

where the processes $I^{\mathbb{N}^{*}}=\left\{I_{t}^{\mathbb{N}^{*}}: t \geq 0\right\}, I^{\mathbb{P}^{*}}=\left\{I_{t}^{\mathbb{P}^{*}}: t \geq 0\right\}$ and $I^{\eta}=\left\{I_{t}^{\eta}: t \geq 0\right\}$, conditioned on $Z$, are independent of each other. We denote the law of $I$ by $\mathbb{Q}_{\nu}$. Recall that $v$ is the initial configuration of $Z$.

For $\mu \in \mathcal{M}_{F}\left(\mathbb{R}^{d}\right)$, let $\widetilde{X}$ be an independent copy of $X$ under $\mathbb{P}_{\mu}^{*}$ and be independent of $I$. Then we define a measure-valued process $\Lambda=\left\{\Lambda_{t}: t \geq 0\right\}$ by

$$
\Lambda=\widetilde{X}+I
$$

Note that $Z, \tilde{X}$ and the three immigration processes above are defined on the same probability space. We denote the law of $\Lambda$ by $\mathbf{P}_{\mu \times v}$. When $v$ is a Poisson random measure with intensity measure $\lambda^{*} \mu$, then we write this law by $\mathbf{P}_{\mu}$. The following result is proved in [9].

Proposition 2.1 For any $\mu \in \mathcal{M}_{F}\left(\mathbb{R}^{d}\right)$, the process $\left(\Lambda, \mathbf{P}_{\mu}\right)$ is Markovian and has the same law as $\left(X, \mathbb{P}_{\mu}\right)$.

We will need the following $\sigma$-fields later on:

$$
\begin{aligned}
& \mathcal{F}_{t}=\sigma\left(\Lambda_{s}, s \leq t\right), \quad t \geq 0, \\
& \mathcal{G}_{t}=\sigma\left(\Lambda_{s}, Z_{s}, s \leq t\right), \quad t \geq 0 .
\end{aligned}
$$

\subsection{Moments}

Now we use Laplace transforms to calculate the moments of $X$. We will omit some details, for these omitted details, see [13]. For any $f \in \mathcal{P}$, we define

$$
u_{f}(x, t, \theta)=-\log \mathbb{P}_{\delta_{x}}\left(e^{-\left\langle\theta f, X_{t}\right\rangle}\right),
$$

then

$$
u_{f}(x, t, \theta)+\Pi_{x} \int_{0}^{t} \psi\left(u_{f}\left(\xi_{s}, t-s, \theta\right)\right) d s=\theta \Pi_{x} f\left(\xi_{t}\right)
$$

For convenience, we use $u_{f}^{(n)}(x, t, 0)$ to denote $\left.\frac{\mathrm{d}^{n} u_{f}(x, t, \theta)}{\mathrm{d} \theta^{n}}\right|_{\theta=0}$. Differentiating both sides of (2.7) with respect to $\theta$, we get

$$
\begin{aligned}
u_{f}^{(1)}(x, t, 0) & =e^{-\psi^{\prime}(0+) t} T_{t} f(x), \\
u_{f}^{(2)}(x, t, 0) & =-\psi^{(2)}(0+) \int_{0}^{t} e^{-\psi^{\prime}(0+)(t-s)} T_{t-s}\left[u_{f}^{(1)}(\cdot, s, 0)\right]^{2}(x) d s \\
& =-A e^{\alpha t} \int_{0}^{t} e^{\alpha s} T_{t-s}\left[T_{s} f\right]^{2}(x) d s .
\end{aligned}
$$


The moments are given by

$$
\mathbb{P}_{\mu}\left(\left\langle f, X_{t}\right\rangle\right)^{n}=\left.(-1)^{n} \frac{\mathrm{d}^{n}\left(e^{-\left\langle u_{f}(\cdot, t, \theta), \mu\right\rangle}\right)}{\mathrm{d} \theta^{n}}\right|_{\theta=0} .
$$

In particular,

$$
\begin{gathered}
\mathbb{P}_{\mu}\left\langle f, X_{t}\right\rangle=\left\langle u_{f}^{(1)}(x, t, 0), \mu\right\rangle=e^{\alpha t}\left\langle T_{t} f, \mu\right\rangle, \\
\mathbb{P}_{\mu}\left(\left\langle f, X_{t}\right\rangle-\mathbb{P}_{\mu}\left\langle f, X_{t}\right\rangle\right)^{2}=-\left\langle u_{f}^{(2)}(x, t, 0), \mu\right\rangle .
\end{gathered}
$$

Recall that $\widetilde{X}_{t}$ is defined in Sect. 2.1. It is a subcritical superprocess with branching mechanism $\psi^{*}(\lambda)=\psi\left(\lambda+\lambda^{*}\right)$. Thus $\frac{\mathrm{d}^{m} \psi^{*}}{\mathrm{~d} \lambda^{m}}(0+)=\frac{\mathrm{d}^{m} \psi}{\mathrm{d} \lambda^{m}}\left(\lambda^{*}\right)$ exists for all $m \geq 1$. For any $f \in \mathcal{P}$, we define

$$
u_{f}^{*}(x, t, \theta)=-\log \mathbb{P}_{\delta_{x}}\left(e^{-\left\langle\theta f, \widetilde{X}_{t}\right\rangle}\right)
$$

Then

$$
u_{f}^{*}(x, t, \theta)+\Pi_{x} \int_{0}^{t} \psi^{*}\left(u_{f}^{*}\left(\xi_{s}, t-s, \theta\right)\right) d s=\theta \Pi_{x} f\left(\xi_{t}\right) .
$$

Differentiating both sides of (2.12) with respect to $\theta$, we have

$$
\begin{aligned}
\left(u_{f}^{*}\right)^{(1)}(x, t, 0)= & e^{-\alpha^{*} t} T_{t} f(x), \\
\left(u_{f}^{*}\right)^{(2)}(x, t, 0)= & -\left(\psi^{*}\right)^{(2)}(0+) \int_{0}^{t} e^{-\left(\psi^{*}\right)^{\prime}(0+)(t-s)} T_{t-s}\left[\left(u_{f}^{*}\right)^{(1)}(\cdot, s, 0)\right]^{2}(x) d s \\
= & -\left(\psi^{*}\right)^{(2)}(0+) e^{-\alpha^{*} t} \int_{0}^{t} e^{-\alpha^{*} s} T_{t-s}\left[T_{s} f\right]^{2}(x) d s, \\
\left(u_{f}^{*}\right)^{(3)}(x, t, 0)= & -\left(\psi^{*}\right)^{(3)}(0+) \int_{0}^{t} e^{-\alpha^{*} s} T_{s}\left[\left(u_{f}^{*}\right)^{(1)}(\cdot, t-s, 0)\right]^{3}(x) d s \\
& -3\left(\psi^{*}\right)^{(2)}(0+) \int_{0}^{t} e^{-\alpha^{*} s} T_{s}\left[\left(\left(u_{f}^{*}\right)^{(1)}\left(u_{f}^{*}\right)^{(2)}\right)(\cdot, t-s, 0)\right](x) d s,
\end{aligned}
$$

and

$$
\left(u_{f}^{*}\right)^{(4)}(x, t, 0)=-\int_{0}^{t} e^{-\alpha^{*} s} T_{s}[J(\cdot, t-s)](x) d s,
$$

where

$$
\begin{aligned}
J(x, t)= & {\left[\left(\psi^{*}\right)^{(4)}(0)\left(\left(u_{f}^{*}\right)^{(1)}\right)^{4}+6\left(\psi^{*}\right)^{(3)}(0)\left(\left(u_{f}^{*}\right)^{(1)}\right)^{2}\left(u_{f}^{*}\right)^{(2)}\right](x, t, 0) } \\
& +\left[4\left(\psi^{*}\right)^{(2)}(0)\left(u_{f}^{*}\right)^{(1)}\left(u_{f}^{*}\right)^{(3)}+3\left(\psi^{*}\right)^{(2)}(0)\left(\left(u_{f}^{*}\right)^{(2)}\right)^{2}\right](x, t, 0) .
\end{aligned}
$$

By (2.12), the moments of $\widetilde{X}$ are given by

$$
\mathbb{P}_{\mu}\left(\left\langle f, \widetilde{X}_{t}\right\rangle\right)^{n}=\left.(-1)^{n} \frac{\mathrm{d}^{n}\left(e^{-\left\langle u_{f}^{*}(\cdot, t, \theta), \mu\right\rangle}\right)}{\mathrm{d} \theta^{n}}\right|_{\theta=0} .
$$


In particular, we have

$$
\begin{aligned}
& \mathbb{P}_{\mu}\left\langle f, \tilde{X}_{t}\right\rangle=\left\langle\left(u_{f}^{*}\right)^{(1)}(x, t, 0), \mu\right\rangle=e^{-\alpha^{*} t}\left\langle T_{t} f, \mu\right\rangle, \\
& \mathbb{P}_{\mu}\left(\left\langle f, \tilde{X}_{t}\right\rangle-\mathbb{P}_{\mu}\left\langle f, \tilde{X}_{t}\right\rangle\right)^{2}=-\left\langle\left(u_{f}^{*}\right)^{(2)}(x, t, 0), \mu\right\rangle, \\
& \mathbb{P}_{\mu}\left(\left\langle f, \tilde{X}_{t}\right\rangle-\mathbb{P}_{\mu}\left\langle f, \tilde{X}_{t}\right\rangle\right)^{4}=-\left\langle\left(u_{f}^{*}\right)^{(4)}(x, t, 0), \mu\right\rangle+3\left\langle\left(u_{f}^{*}\right)^{(2)}(x, t, 0), \mu\right\rangle^{2} .
\end{aligned}
$$

\subsection{Estimates on the Semigroup $T_{t}$}

Recall that $\xi=\left\{\xi_{t}: t \geq 0\right\}$ is the OU process and $\left\{T_{t}\right\}$ is the semigroup of $\xi$. It is well-known that under $\Pi_{x}, \xi_{t} \sim \mathcal{N}\left(x e^{-b t}, \sigma_{t}^{2}\right)$, where $\sigma_{t}^{2}=\sigma^{2}\left(1-e^{-2 b t}\right) /(2 b)$. Let $G$ be an $\mathbb{R}^{d}$-valued standard normal random variable, then using $(a+b)^{n} \leq 2^{n}\left(a^{n}+b^{n}\right), a \geq 0, b \geq 0$, we get

$$
T_{t}\left(\|\cdot\|^{n}\right)(x)=E\left(\left\|\sigma_{t} G+x e^{-b t}\right\|^{n}\right) \leq 2^{n}\left[(\sigma / \sqrt{2 b})^{n} E\left(\|G\|^{n}\right)+\|x\|^{n}\right] .
$$

Using this, we can easily get that

$$
T_{t}\left(1+\|\cdot\|^{n}\right)(x) \leq c(n)\left(1+\|x\|^{n}\right),
$$

where $c(n)$ does not depend on $t$.

Lemma 2.2 For any $f \in L^{2}(\varphi)$, we have that, for every $x \in \mathbb{R}^{d}$,

$$
\begin{aligned}
& T_{t} f(x)=\sum_{n=\gamma(f)}^{\infty} e^{-n b t} \sum_{|p|=n} a_{p} \phi_{p}(x), \\
& \lim _{t \rightarrow \infty} e^{\gamma(f) b t} T_{t} f(x)=\sum_{|p|=\gamma(f)} a_{p} \phi_{p}(x) .
\end{aligned}
$$

Moreover, there exists $c>0$ such that for $t \geq 1$,

$$
\left|T_{t} f(x)\right| \leq c e^{-\gamma(f) b t} e^{\frac{b}{2 \sigma^{2}}\|x\|^{2}}, \quad x \in \mathbb{R}^{d} .
$$

Proof For every $f \in L^{2}(\varphi)$, using the fact that $\varphi(x)$ is the invariant density of $\xi$ we get that

$$
\int \varphi(x)\left(T_{t}|f|(x)\right)^{2} d x \leq \int \varphi(x) T_{t}\left[|f|^{2}\right](x) d x=\int|f(y)|^{2} \varphi(y) d y<\infty,
$$

so $T_{t} f(x) \in L^{2}(\varphi)$. Moreover, by the fact $\xi_{t} \sim \mathcal{N}\left(x e^{-b t}, \sigma_{t}^{2}\right), T_{t}|f|(x)$ is continuous in $x$. Thus $T_{t}|f|(x)<\infty$ for all $x \in \mathbb{R}^{d}$. (2.26) implies that $T_{t}$ is a bounded linear operator on $L^{2}(\varphi)$. Let $f_{k}(x)=\sum_{n=0}^{k} \sum_{|p|=n} a_{p} \phi_{p}(x)$. Since $f_{k} \rightarrow f$ in $L^{2}(\varphi)$, we have $T_{t} f_{k} \rightarrow T_{t} f$ in $L^{2}(\varphi)$, as $k \rightarrow \infty$. By linearity, we have

$$
T_{t} f_{k}(x)=\sum_{n=0}^{k} e^{-n b t}\left(\sum_{|p|=n} a_{p} \phi_{p}(x)\right) .
$$

We claim that the series $\sum_{n=0}^{\infty} e^{-n b t}\left(\sum_{|p|=n} a_{p} \phi_{p}(x)\right)$ is uniformly convergent on any compact subset of $\mathbb{R}^{d}$. Thus $\sum_{n=0}^{\infty} e^{-n b t}\left(\sum_{|p|=n} a_{p} \phi_{p}(x)\right)$ is continuous in $x$. So for all $x \in \mathbb{R}^{d}$,

$$
T_{t} f(x)=\sum_{n=0}^{\infty} e^{-n b t}\left(\sum_{|p|=n} a_{p} \phi_{p}(x)\right) .
$$


Now we prove the claim. In fact, by Cramer's inequality (for example, see [19, Equation (19) on p. 207]), for all $p \in \mathbb{Z}_{+}^{d}$ we have

$$
\left|\phi_{p}(x)\right| \leq K e^{\frac{b}{2 \sigma^{2}}\|x\|^{2}},
$$

where $K$ is a constant. So we only need to prove $\sum_{n=0}^{\infty} e^{-n b t}\left(\sum_{|p|=n}\left|a_{p}\right|\right)<\infty$. By Hölder's inequality,

$$
\sum_{n=0}^{\infty} e^{-n b t}\left(\sum_{|p|=n}\left|a_{p}\right|\right) \leq\left(\sum_{n=\gamma(f)}^{\infty} K_{n} e^{-2 n b t}\right)^{1 / 2}\left(\sum_{n=\gamma(f)}^{\infty} \sum_{|p|=n}\left|a_{p}\right|^{2}\right)^{1 / 2}
$$

where $K_{n}=\left(\begin{array}{c}n+d-1 \\ d-1\end{array}\right)=\sharp\left\{p \in \mathbb{Z}_{+}^{d}:|p|=n\right\}$. Since $K_{n} \leq(n+d)^{d}$, we have that

$$
\sum_{n=\gamma(f)}^{\infty} K_{n} e^{-2 n b t}<\infty
$$

Using the fact that $\left\{\phi_{p}(x), p \in \mathbb{Z}_{+}^{d}\right\}$ form a complete orthogonal basis for $L^{2}(\varphi)$, we get $\sum_{n=\gamma(f)}^{\infty} \sum_{|p|=n}\left|a_{p}\right|^{2}=\int \varphi(x)|f(x)|^{2} d x<\infty$. Therefore the claim is true.

By (2.27) and (2.28), for $t \geq 1$, we have

$$
\begin{aligned}
\left|T_{t} f(x)\right| & \leq e^{-\gamma(f) b t}\left(\sum_{n=0}^{\infty} K_{n+\gamma(f)} e^{-2 n b}\right)^{1 / 2}\left(\sum_{n=\gamma(f)}^{\infty} \sum_{|p|=n}\left|a_{p}\right|^{2}\right)^{1 / 2} K e^{\frac{b}{2 \sigma^{2}}\|x\|^{2}} \\
& \lesssim e^{-\gamma(f) b t} e^{\frac{b}{2 \sigma^{2}}\|x\|^{2}}, \quad x \in \mathbb{R}^{d}
\end{aligned}
$$

Therefore, for $t \geq 1$,

$$
\begin{aligned}
\left|e^{\gamma(f) b t} T_{t} f(x)-\sum_{|p|=\gamma(f)} a_{p} \phi_{p}(x)\right| & =e^{\gamma(f) b t}\left|T_{t} f(x)-e^{-\gamma(f) b t} \sum_{|p|=\gamma(f)} a_{p} \phi_{p}(x)\right| \\
& =e^{\gamma(f) b t}\left|T_{t}\left(f-\sum_{|p|=\gamma(f)} a_{p} \phi_{p}\right)(x)\right| \\
& \lesssim e^{-b t} e^{\frac{b}{2 \sigma^{2}}\|x\|^{2}},
\end{aligned}
$$

which implies (2.24). The proof is now complete.

For $p \in \mathbb{Z}_{+}^{d}$, we use the notation $f^{(p)}(x):=\frac{\partial}{\partial x_{1}^{p_{1}} \partial x_{2}^{p_{2} \ldots \partial x_{d}^{p_{d}}}} f(x)$. Define

$$
\mathcal{P}^{*}=\left\{f \in C^{\infty}: f^{(p)} \in \mathcal{P} \text { for all } p \in \mathbb{Z}_{+}^{d}\right\}
$$

It can be easily shown that, for any $f \in \mathcal{P}, T_{t} f(x) \in \mathcal{P}^{*}$ for every $t>0$.

Lemma 2.3 For any $f \in \mathcal{P}^{*}$ and $p \in \mathbb{Z}_{+}^{d}$ satisfying $0 \leq|p| \leq \gamma(f)$, we have $\gamma\left(f^{(p)}\right) \geq$ $\gamma(f)-|p|$. 
Proof By the definition of $\phi_{p}$ and $\varphi$, it is easy to check that

$$
\phi_{p}(x) \varphi(x)=(-1)^{|p|} c_{p} \varphi^{(p)}(x),
$$

where $c_{p}=\frac{1}{\sqrt{p ! 2|p|}}\left(\frac{\sigma^{2}}{b}\right)^{|p| / 2}$. Integrating by parts, we get

$$
\int f(x) \phi_{p}(x) \varphi(x) d x=c_{p} \int_{\mathbb{R}^{d}} f^{(p)}(x) \varphi(x) d x
$$

Thus

$$
\gamma(f)=\inf \left\{k: \text { there exists } p \text { such that }|p|=k \text { and } \int_{\mathbb{R}^{d}} f^{(p)}(x) \varphi(x) d x \neq 0\right\} .
$$

Hence if $\left|p^{\prime}\right|<\gamma(f)-|p|$, we have $\int_{\mathbb{R}^{d}} f^{\left(p+p^{\prime}\right)}(x) \varphi(x) d x=0$, which implies $\gamma\left(f^{(p)}\right) \geq$ $\gamma(f)-|p|$. later.

In the following lemma, we give another estimate for $T_{t} f$, which will be very useful

Lemma 2.4 For every $f \in \mathcal{P}$, there exist $r \in \mathbb{N}$ and $c>0$ such that

$$
\begin{aligned}
& e^{\gamma(f) b t}\left|T_{t} f(x)\right| \leq c\left(1+\|x\|^{r}\right), \\
& \left|e^{\gamma(f) b t} T_{t} f(x)-\sum_{|p|=\gamma(f)} a_{p} \phi_{p}(x)\right| \leq c e^{-b t}\left(1+\|x\|^{r}\right) .
\end{aligned}
$$

Proof Let $g(x)=T_{1} f(x) \in \mathcal{P}^{*}$. Then $\gamma(g)=\gamma(f)$ and there exist $k \in \mathbb{N}$ and $c_{1}>0$ such that, for $|p|=0,1, \ldots, \gamma(f),\left|g^{(p)}(x)\right| \leq c_{1}\left(1+\|x\|^{k}\right)$. For $x=\left(x_{1}, x_{2}, \ldots, x_{d}\right) \in \mathbb{R}^{d}$, we define $x^{p}:=\prod_{i=1}^{d} x_{i}^{p_{i}}$. Then for $s>0$ we have

$$
\begin{aligned}
T_{s} g(x)= & T_{s}\left[g\left(\cdot+x e^{-b s}\right)\right](0) \\
= & T_{s}\left[g\left(\cdot+x e^{-b s}\right)-\sum_{m=0}^{\gamma(f)-1} \sum_{|p|=m} g^{(p)}(\cdot) x^{p} e^{-m b s} / p !\right](0) \\
& +\sum_{m=0}^{\gamma(f)-1} \sum_{|p|=m} T_{s}\left[g^{(p)}\right](0) x^{p} e^{-m b s} / p ! \\
= & (I)+(I I) .
\end{aligned}
$$

It follows from (2.25) and the fact that $\gamma\left(g^{(p)}\right) \geq \gamma(g)-|p|$, we have

$$
\sup _{s>0} e^{(\gamma(g)-|p|) b s}\left|T_{s}\left[g^{(p)}\right](0)\right|<\infty .
$$

Thus

$$
|(I I)| \lesssim e^{-\gamma(f) b s} \sum_{m=0}^{\gamma(f)-1} \sum_{|p|=m}\left|x^{p}\right| \lesssim e^{-\gamma(f) b s}\left(1+\|x\|^{\gamma(f)}\right) .
$$


Using Taylor's formula and the fact $\left|g^{(p)}(x)\right| \lesssim 1+\|x\|^{k}$, we get

$$
\begin{aligned}
\left|g\left(y+x e^{-b s}\right)-\sum_{m=0}^{\gamma(f)-1} \sum_{|p|=m} g^{(p)}(y) x^{p} e^{-m b s} / p !\right| & =\sum_{|p|=\gamma(f)}\left|g^{(p)}(\theta) \| x^{p}\right| e^{-\gamma(f) b s} /(\gamma(f) !) \\
& \lesssim\left(1+\|y\|^{k}+\|x\|^{k}\right)|x|^{\gamma(f)} e^{-\gamma(f) b s},
\end{aligned}
$$

where $\theta$ is a point on the line segment connecting $y$ and $y+x e^{-b s}$. Then by the fact that $T_{s}\left[\|\cdot\|^{k}\right](x) \lesssim 1+\|x\|^{k}$, we get $\sup _{s>0} T_{s}\left[\|\cdot\|^{k}\right](0)<\infty$. Therefore, we have

$$
|(I)| \lesssim\left(1+\|x\|^{k+\gamma(f)}\right) e^{-\gamma(f) b s} .
$$

Consequently,

$$
e^{\gamma(f) b s}\left|T_{s} g\right|(x) \lesssim 1+\|x\|^{k+\gamma(f)} .
$$

Let $r_{1}=k+\gamma(f)$. For $t \geq 1$, combining $T_{t} f(x)=T_{t-1}(g)(x)$ with the above inequality, we arrive at (2.32) for $t \geq 1$. For $t<1$,

$$
e^{\gamma(f) b t}\left|T_{t} f(x)\right| \lesssim e^{\gamma(f) b}\left(1+\|x\|^{k}\right) \lesssim 1+\|x\|^{r_{1}},
$$

so (2.32) is also valid.

It follows from (2.32) that there exists $r_{2} \in \mathbb{N}$ such that

$$
e^{(\gamma(f)+1) b t}\left|T_{t} f(x)-e^{-\gamma(f) b t} \sum_{|p|=\gamma(f)} a_{p} \phi_{p}(x)\right| \lesssim 1+\|x\|^{r_{2}} .
$$

Now (2.33) follows immediately.

From the above calculations, we have

Lemma 2.5 Let $f \in \mathcal{P}$.

(i) If $\alpha<2 \gamma(f) b$, then

$$
\begin{aligned}
\lim _{t \rightarrow \infty} e^{-(\alpha / 2) t} \mathbb{P}_{\delta_{x}}\left(\left\langle f, X_{t}\right\rangle\right) & =0, \\
\lim _{t \rightarrow \infty} e^{-\alpha t} \mathbb{V a r} r_{\delta_{x}}\left\langle f, X_{t}\right\rangle & =\sigma_{f}^{2},
\end{aligned}
$$

where $\mathbb{V}_{\delta_{\delta_{x}}}$ stands for the variance under $\mathbb{P}_{\delta_{x}}$ and $\sigma_{f}^{2}$ is defined in (1.14).

(ii) If $\alpha=2 \gamma(f) b$, then

$$
\lim _{t \rightarrow \infty} t^{-1 / 2} e^{-(\alpha / 2) t} \mathbb{P}_{\delta_{x}}\left(\left\langle f, X_{t}\right\rangle\right)=0,
$$

and there exists $r \in \mathbb{N}$ such that

$$
\left|t^{-1} e^{-\alpha t} \operatorname{Var}_{\delta_{x}}\left\langle f, X_{t}\right\rangle\right| \lesssim 1+\|x\|^{2 r}
$$

and

$$
\left|t^{-1} e^{-\alpha t} \operatorname{Var}_{\delta_{x}}\left\langle f, X_{t}\right\rangle-\rho_{f}^{2}\right| \lesssim t^{-1}\left(1+\|x\|^{r}\right)
$$


which in particular implies that

$$
\lim _{t \rightarrow \infty} t^{-1} e^{-\alpha t} \operatorname{Var}_{\delta_{x}}\left\langle f, X_{t}\right\rangle=\rho_{f}^{2},
$$

where $\rho_{f}^{2}$ is defined in (1.17).

(iii) If $\alpha>2 \gamma(f) b$, then

$$
\lim _{t \rightarrow \infty} e^{-2(\alpha-\gamma(f) b) t} \operatorname{Var}_{\delta_{x}}\left\langle f, X_{t}\right\rangle=\eta_{f}^{2}(x),
$$

where

$$
\eta_{f}^{2}(x)=A \int_{0}^{\infty} e^{-(\alpha-2 \gamma(f) b) s} T_{s}\left(\sum_{|p|=\gamma(f)} a_{p} \phi_{p}\right)^{2}(x) d s .
$$

Proof It follows from (2.9) and (2.11) that

$$
\operatorname{Var}_{\delta_{x}}\left\langle f, X_{t}\right\rangle=A e^{\alpha t} \int_{0}^{t} e^{\alpha s} T_{t-s}\left[T_{s} f\right]^{2}(x) d s=A e^{2 \alpha t} \int_{0}^{t} e^{-\alpha s} T_{s}\left[T_{t-s} f\right]^{2}(x) d s .
$$
Thus

(i) If $\alpha<2 \gamma(f) b$, by Lemma 2.2, we have $\lim _{t \rightarrow \infty} e^{\gamma(f) b t} T_{t} f(x)=\sum_{|p|=\gamma(f)} a_{p} \phi_{p}(x)$.

$$
\lim _{t \rightarrow \infty} e^{-(\alpha / 2) t} \mathbb{P}_{\delta_{x}}\left\langle f, X_{t}\right\rangle=\lim _{t \rightarrow \infty} e^{(\alpha-2 \gamma(f) b) t / 2}\left[e^{\gamma(f) b t} T_{t} f(x)\right]=0 .
$$

It follows from Lemma 2.4 that there exists $r \in \mathbb{N}$ such that $e^{\gamma(f) b s}\left|T_{s} f\right|(x) \lesssim 1+\|x\|^{r}$. Using (2.22), we have

$$
T_{t-s}\left[e^{\gamma(f) b s} T_{s} f\right]^{2}(x) \lesssim 1+\|x\|^{2 r} .
$$

Thus $e^{\alpha s} T_{t-s}\left[T_{s} f\right]^{2}(x) \lesssim e^{(\alpha-2 \gamma(f) b) s}\left(1+\|x\|^{2 r}\right)$. Hence by the dominated convergence theorem, we get

$$
\lim _{t \rightarrow \infty} \int_{0}^{t} e^{\alpha s} T_{t-s}\left[T_{s} f\right]^{2}(x) d s=\int_{0}^{\infty} e^{\alpha s}\left\langle\left(T_{s} f\right)^{2}, \varphi\right\rangle d s .
$$

By (2.23) and the fact that $\left\{\phi_{p}(x), p \in \mathbb{Z}_{+}^{d}\right\}$ is orthonormal in $L^{2}(\varphi)$, we have

$$
\left\langle\left(T_{s} f\right)^{2}, \varphi\right\rangle=\sum_{n=\gamma(f)}^{\infty} \sum_{|p|=n} e^{-2 n b s} a_{p}^{2}
$$

which implies (2.34).

(ii) If $\alpha=2 \gamma(f) b$, then by (2.41), we have

$$
t^{-1} e^{-\alpha t} \operatorname{Var}_{\delta_{x}}\left\langle f, X_{t}\right\rangle=A t^{-1} \int_{0}^{t} T_{t-s}\left[e^{\gamma(f) b s} T_{s} f\right]^{2}(x) d s .
$$

By Lemma 2.4, there exists $r \in \mathbb{N}$ satisfying (2.32), (2.33) and

$$
\left|\sum_{|p|=\gamma(f)} a_{p} \phi_{p}(x)\right| \lesssim 1+\|x\|^{r}
$$


which follows from the fact that $\phi_{p}(x)$ is a polynomial. Then by (2.32) and (2.43), it is easy to get (2.36).

Let $h(x):=\left(\sum_{|p|=\gamma(f)} a_{p} \phi_{p}(x)\right)^{2}$. Then we have

$$
\begin{aligned}
& \left|\left(e^{\gamma(f) b s} T_{s} f(x)\right)^{2}-h(x)\right| \\
& \quad \leq\left|e^{\gamma(f) b s} T_{s} f(x)-\sum_{|p|=\gamma(f)} a_{p} \phi_{p}(x)\right|\left(e^{\gamma(f) b s}\left|T_{s} f\right|(x)+\left|\sum_{|p|=\gamma(f)} a_{p} \phi_{p}(x)\right|\right) \\
& \quad \lesssim e^{-b s}\left(1+\|x\|^{2 r}\right) .
\end{aligned}
$$

Since $\gamma(h)=0$ and $\sum_{|p|=\gamma(f)} a_{p}^{2}=\langle h, \varphi\rangle$, by (2.33), there exists $r^{\prime} \in \mathbb{N}$ such that

$$
\left|T_{t-s} h(x)-\sum_{|p|=\gamma(f)} a_{p}^{2}\right| \lesssim e^{-b(t-s)}\left(1+\|x\|^{r^{\prime}}\right) .
$$

Let $r_{0}=\max \left(2 r, r^{\prime}\right)$, then

$$
\begin{aligned}
& \left|T_{t-s}\left(e^{\gamma(f) b s} T_{s} f\right)^{2}(x)-\sum_{|p|=\gamma(f)} a_{p}^{2}\right| \\
& \quad \leq T_{t-s}\left|\left(e^{\gamma(f) b s} T_{s} f(x)\right)^{2}-h(x)\right|+\left|T_{t-s} h(x)-\sum_{|p|=\gamma(f)} a_{p}^{2}\right| \\
& \quad \lesssim\left(e^{-b s}+e^{-b(t-s)}\right)\left(1+\|x\|^{r_{0}}\right) .
\end{aligned}
$$

It follows that

$$
\begin{aligned}
& \frac{1}{t} \int_{0}^{t}\left|T_{t-s}\left(e^{\gamma(f) b s} T_{s} f\right)^{2}(x)-\sum_{|p|=\gamma(f)} a_{p}^{2}\right| d s \\
& \quad \lesssim \frac{\int_{0}^{t}\left(e^{-b s}+e^{-b(t-s)}\right)\left(1+\|x\|^{r_{0}}\right) d s}{t} \lesssim t^{-1}\left(1+\|x\|^{r_{0}}\right) .
\end{aligned}
$$

Then (2.37) follows from (2.43) and (2.45).

(iii) If $\alpha>2 \gamma(f) b$, then by (2.41), we have

$$
e^{-2(\alpha-\gamma(f) b) t} \mathbb{V} a r_{\delta_{x}}\left\langle f, X_{t}\right\rangle=A \int_{0}^{t} e^{-(\alpha-2 \gamma(f) b) s} T_{s}\left[e^{\gamma(f) b(t-s)} T_{t-s} f\right]^{2}(x) d s .
$$

By Lemma 2.4, there exists $r \in \mathbb{N}$ such that $\left[e^{\gamma(f) b(t-s)} T_{t-s} f(x)\right]^{2} \leq c\left(1+\|x\|^{2 r}\right)$. Thus

$$
T_{s}\left[e^{\gamma(f) b(t-s)} T_{t-s} f\right]^{2}(x) \lesssim 1+\|x\|^{2 r} .
$$

Now by the dominated convergence theorem and (2.24), we have

$$
\begin{aligned}
& \lim _{t \rightarrow \infty} \int_{0}^{t} e^{-(\alpha-2 \gamma(f) b) s} T_{s}\left[e^{\gamma(f) b(t-s)} T_{t-s} f\right]^{2}(x) d s \\
& \quad=A \int_{0}^{\infty} e^{-(\alpha-2 \gamma(f) b) s} T_{s}\left(\sum_{|p|=\gamma(f)} a_{p} \phi_{p}\right)^{2}(x) d s .
\end{aligned}
$$

The proof of (iii) is now complete. 
According to [9], under $\mathbf{P}_{\delta_{x}}$, we have that, conditioned on $\mathcal{F}_{t}$ (see (2.5)), the backbone $Z_{t}$ is a Poisson point process with the intensity $\lambda^{*} \Lambda_{t}$. In particular, $Z_{0}=N \delta_{x}$, where $N$ is a Poisson random variable with parameter $\lambda^{*}$. Then we have

$$
\Lambda_{t}=\widetilde{X}_{t}+\sum_{j=1}^{N} I_{t}^{j}
$$

where $I^{j}, j=1,2, \ldots$ are independent copies of $I$ under $\mathbb{Q}_{\delta_{x}}$ and are independent of $N$. The first moment of $I$ can be calculated by

$$
\mathbf{P}_{\delta_{x}}\left\langle f, \Lambda_{t}\right\rangle=\mathbf{P}_{\delta_{x}}\left\langle f, \tilde{X}_{t}\right\rangle+\lambda^{*} \mathbb{Q}_{\delta_{x}}\left\langle f, I_{t}\right\rangle
$$

Thus

$$
\mathbb{Q}_{\delta_{x}}\left\langle f, I_{t}\right\rangle=\frac{1}{\lambda^{*}}\left(\mathbf{P}_{\delta_{x}}\left\langle f, \Lambda_{t}\right\rangle-\mathbf{P}_{\delta_{x}}\left\langle f, \tilde{X}_{t}\right\rangle\right)=\frac{1}{\lambda^{*}}\left(e^{\alpha t}-e^{-\alpha^{*} t}\right) T_{t} f(x) .
$$

For the second moment, let $\mathbf{V} a r_{\delta_{x}}$ stand for the variance under $\mathbf{P}_{\delta_{x}}$ and $\mathbb{V}_{\delta_{x}}$ stand for the variance under $\mathbb{Q}_{\delta_{x}}$. By (2.46), we have

$$
\operatorname{Var}_{\delta_{x}}\left\langle f, \Lambda_{t}\right\rangle=\mathbf{V}_{\delta_{\delta_{x}}}\left\langle f, \widetilde{X}_{t}\right\rangle+\lambda^{*} \mathbb{Q}_{\delta_{x}}\left\langle f, I_{t}\right\rangle^{2}
$$

Thus

$$
\mathbb{Q}_{\delta_{x}}\left\langle f, I_{t}\right\rangle^{2}=\frac{1}{\lambda^{*}}\left(\operatorname{Var}_{\delta_{x}}\left\langle f, X_{t}\right\rangle-\operatorname{Var}_{\delta_{x}}\left\langle f, \widetilde{X}_{t}\right\rangle\right)
$$

Corollary 2.6 Let $\left\{I_{t}\right\}_{t \geq 0}$ be the process described in the Sect. 2.1 and $f \in \mathcal{P}$.

(i) If $\alpha<2 \gamma(f) b$, then

$$
\begin{aligned}
& \lim _{t \rightarrow \infty} e^{-(\alpha / 2) t} \mathbb{Q}_{\delta_{x}}\left(\left\langle f, I_{t}\right\rangle\right)=0, \\
& \lim _{t \rightarrow \infty} e^{-\alpha t} \mathbb{V}_{\delta_{x}}\left\langle f, I_{t}\right\rangle=\frac{A}{\lambda^{*}} \int_{0}^{\infty} e^{\alpha s}\left\langle\left(T_{s} f\right)^{2}, \varphi\right\rangle d s=\frac{\sigma_{f}^{2}}{\lambda^{*}} .
\end{aligned}
$$

(ii) If $\alpha=2 \gamma(f) b$, then

$$
\lim _{t \rightarrow \infty} t^{-1 / 2} e^{-(\alpha / 2) t} \mathbb{Q}_{\delta_{x}}\left(\left\langle f, I_{t}\right\rangle\right)=0,
$$

and there exists $r \in \mathbb{N}$ such that

$$
\left|t^{-1} e^{-\alpha t} \mathbb{V}_{\delta_{x}}\left\langle f, I_{t}\right\rangle\right| \lesssim 1+\|x\|^{2 r}
$$

and

$$
\left|t^{-1} e^{-\alpha t} \mathbb{V}_{\delta_{x}}\left\langle f, I_{t}\right\rangle-\frac{A}{\lambda^{*}} \sum_{|p|=\gamma(f)} a_{p}^{2}\right| \lesssim t^{-1}\left(1+\|x\|^{r}\right),
$$

which in particular implies that

$$
\lim _{t \rightarrow \infty} t^{-1} e^{-\alpha t} \mathbb{V}_{\delta_{x}}\left\langle f, I_{t}\right\rangle=\frac{A}{\lambda^{*}} \sum_{|p|=\gamma(f)} a_{p}^{2} .
$$


(iii) If $\alpha>2 \gamma(f) b$, then

$$
\lim _{t \rightarrow \infty} e^{-2(\alpha-\gamma(f) b) t} \mathbb{V}_{\delta_{x}}\left\langle f, I_{t}\right\rangle=\frac{\eta_{f}^{2}(x)}{\lambda^{*}}-\frac{1}{\left(\lambda^{*}\right)^{2}}\left(\sum_{|p|=\gamma(f)} a_{p} \phi_{p}(x)\right)^{2} .
$$

Proof Using (2.46) and Lemma 2.5, we can easily obtain the corollary. Here we just give the proof of (2.51). By (2.49), we have

$$
e^{-\alpha t} \mathbb{V}_{\delta_{x}}\left\langle f, I_{t}\right\rangle=\frac{1}{\lambda^{*}} e^{-\alpha t} \operatorname{Var}_{\delta_{x}}\left\langle f, X_{t}\right\rangle-\frac{1}{\lambda^{*}} e^{-\alpha t} \mathbf{V} a r_{\delta_{x}}\left\langle f, \tilde{X}_{t}\right\rangle-e^{-\alpha t}\left(\mathbb{Q}_{\delta_{x}}\left\langle f, I_{t}\right\rangle\right)^{2}
$$

Using (2.15) and (2.19), we have

$$
\operatorname{Var}_{\delta_{x}}\left\langle f, \widetilde{X}_{t}\right\rangle=\left(\psi^{*}\right)^{\prime \prime}(0+) e^{-\alpha^{*} t} \int_{0}^{t} e^{-\alpha^{*} s} T_{t-s}\left[T_{s} f\right]^{2}(x) d s .
$$

By the fact that there exists $r \in \mathbb{N}$ such that $\left|T_{t} f(x)\right| \lesssim 1+\|x\|^{r}$, we get $T_{t-s}\left[T_{s} f\right]^{2}(x) \lesssim$ $\left(1+\|x\|^{2 r}\right)$. Thus

$$
\mathbf{V} a r_{\delta_{x}}\left\langle f, \tilde{X}_{t}\right\rangle \lesssim e^{-\alpha^{*} t}\left(1+\|x\|^{2 r}\right) \rightarrow 0, \quad t \rightarrow \infty .
$$

By (2.48), $\left|\mathbb{Q}_{\delta_{x}}\left\langle f, I_{t}\right\rangle\right| \lesssim e^{\alpha t}\left|T_{t} f(x)\right| \lesssim e^{(\alpha-\gamma(f) b) t}\left(1+\|x\|^{r}\right)$, thus we have

$$
\lim _{t \rightarrow \infty} e^{-\alpha t}\left(\mathbb{Q}_{\delta_{x}}\left\langle f, I_{t}\right\rangle\right)^{2} \lesssim \lim _{t \rightarrow \infty} e^{(\alpha-2 \gamma(f) b) t}\left(1+\|x\|^{2 r}\right)=0 .
$$

Now, using (2.34), (2.59) and (2.60), we easily get (2.51).

Lemma 2.7 For $f \in \mathcal{P}$, it holds that

$$
\mathbb{P}_{\mu}\left(\left\langle f, \widetilde{X}_{t}\right\rangle-\mathbb{P}_{\mu}\left\langle f, \widetilde{X}_{t}\right\rangle\right)^{4} \lesssim\left\langle 1+\|x\|^{4 r}, \mu\right\rangle+\left\langle 1+\|x\|^{2 r}, \mu\right\rangle^{2} .
$$

Proof By (2.32), there exists $r \in \mathbb{N}$ such that $\left|T_{t} f(x)\right| \lesssim 1+\|x\|^{r}$. So by (2.14), $\left|\left(u_{f}^{*}\right)^{(1)}(x, t, 0)\right| \lesssim 1+\|x\|^{r}$. By (2.59) and (2.19), we have $\left|\left(u_{f}^{*}\right)^{(2)}(x, t, 0)\right| \lesssim 1+\|x\|^{2 r}$. Thus using (2.16), we get $\left|\left(u_{f}^{*}\right)^{(3)}(x, t, 0)\right| \lesssim 1+\|x\|^{3 r}$. Then by (2.17), we have $\left|\left(u_{f}^{*}\right)^{(4)}(x, t, 0)\right| \lesssim 1+\|x\|^{4 r}$. Now (2.61) follows immediately from (2.20).

\section{Proofs of the Main Theorems}

In this section, we will prove the main results of this paper. Recall that we assume that the initial measure $\mu$ is a finite measure on $\mathbb{R}^{d}$ with compact support, and that $\left(X_{t}, \mathbb{P}_{\mu}\right)$ and $\left(\Lambda_{t}, \mathbf{P}_{\mu}\right)$ have the same law. Thus in the remainder of this paper, we will replace $\left(X_{t}, \mathbb{P}_{\mu}\right)$ by $\left(\Lambda_{t}, \mathbf{P}_{\mu}\right)$. Define

$$
\mathcal{L}_{t}=\left\{u \in \mathcal{T}, \tau_{u} \leq t<\sigma_{u}\right\}, \quad t \geq 0,
$$

which is the collection of particles which are alive at time $t$. From the construction of $\Lambda_{t}$, we have

$$
\Lambda_{(t+s)}=\tilde{X}_{s}^{t}+\sum_{u \in \mathcal{L}_{t}} I_{s}^{u, t}
$$


where, conditioned on $\mathcal{G}_{t}, \widetilde{X}^{t}$ is a superprocess with the same law as $X$ under $\mathbb{P}_{\Lambda_{t}^{*}}^{\text {and } I^{u, t}}$ has the same law as $I$ under $\mathbb{Q}_{z_{u}(t)}$. The processes $I^{u, t}, u \in \mathcal{L}_{t}$, are independent.

3.1 The Large Rate Case: $\alpha>2 b \gamma(f)$

Recall that

$$
H_{t}^{p}=e^{-(\alpha-|p| b) t}\left\langle\phi_{p}, X_{t}\right\rangle, \quad t \geq 0 .
$$

Lemma 3.1 $H_{t}^{p}$ is a martingale under $\mathbb{P}_{\mu}$. Moreover, if $\alpha>2|p| b$, we have $\sup _{t} \mathbb{P}_{\mu}\left(H_{t}^{p}\right)^{2}<$ $\infty$, and therefore the limit

$$
H_{\infty}^{p}:=\lim _{t \rightarrow \infty} H_{t}^{p}
$$

exists $\mathbb{P}_{\mu}$-a.s. and in $L^{2}\left(\mathbb{P}_{\mu}\right)$.

Proof Since $\phi_{p}$ is an eigenfunction of $L$ corresponding to $-|p| b$, by (2.10), we have $\mathbb{P}_{\mu} H_{t}^{p}=\left\langle\phi_{p}, \mu\right\rangle$. Thus, by the Markov property, we get that $H_{t}^{p}$ is a martingale. Using (2.10) and (2.11), we get

$$
\mathbb{P}_{\mu}\left\langle\phi_{p}, X_{t}\right\rangle^{2}=e^{2(\alpha-|p| b) t}\left\langle\phi_{p}, \mu\right\rangle^{2}+A e^{\alpha t} \int_{\mathbb{R}^{d}} \int_{0}^{t} e^{(\alpha-2|p| b) s} T_{t-s}\left[\phi_{p}^{2}\right](x) d s \mu(d x) .
$$

Thus, when $\alpha>2|p| b$, we have by the definition of $H_{t}^{p}$,

$$
\begin{aligned}
\mathbb{P}_{\mu}\left(H_{t}^{p}\right)^{2} & =\left\langle\phi_{p}, \mu\right\rangle^{2}+A \int_{\mathbb{R}^{d}} \int_{0}^{t} e^{-(\alpha-2|p| b) s} T_{s}\left[\phi_{p}^{2}\right](x) d s \mu(d x) \\
& \leq\left\langle\phi_{p}, \mu\right\rangle^{2}+A \int_{\mathbb{R}^{d}} \int_{0}^{\infty} e^{-(\alpha-2|p| b) s} T_{s}\left[\phi_{p}^{2}\right](x) d s \mu(d x) .
\end{aligned}
$$

Since $\left|\phi_{p}^{2}\right| \lesssim 1+\|x\|^{2|p|}$, by (2.22), we have $\left|T_{s}\left[\phi_{p}^{2}\right](x)\right| \lesssim 1+\|x\|^{2|p|}$. Thus

$$
\int_{\mathbb{R}^{d}} \int_{0}^{\infty} e^{-(\alpha-2|p| b) s} T_{s}\left[\phi_{p}^{2}\right](x) d s \mu(d x) \lesssim \int_{\mathbb{R}^{d}}\left(1+\|x\|^{2|p|}\right) \mu(d x)<\infty,
$$

from which the convergence asserted in the lemma follows easily.

We now present the proof of Theorem 1.1.

Proof of Theorem 1.1 Define $M_{t}:=e^{-(\alpha-\gamma(f) b) t}\left\langle\tilde{f}, X_{t}\right\rangle$, where

$$
\tilde{f}(x)=f(x)-\sum_{|p|=\gamma(f)} a_{p} \phi_{p}(x)=\sum_{n=\gamma(f)+1}^{\infty} \sum_{|p|=n} a_{p} \phi_{p}(x) .
$$

It is clear that $\gamma(\tilde{f}) \geq \gamma(f)+1$. From Lemma 2.5 and (2.32), we have

(1) If $\alpha>2 \gamma(\tilde{f}) b$, then

$$
\lim _{t \rightarrow \infty} e^{-2(\alpha-\gamma(\tilde{f}) b) t} \mathbb{P}_{\mu}\left\langle\tilde{f}, X_{t}\right\rangle^{2}
$$


exists, thus we have

$$
\begin{aligned}
\mathbb{P}_{\mu} M_{t}^{2} & =e^{-2(\gamma(\tilde{f})-\gamma(f)) b t} e^{-2(\alpha-\gamma(\tilde{f}) b) t} \mathbb{P}_{\mu}\left\langle\tilde{f}, X_{t}\right\rangle^{2} \\
& =O\left(e^{-2(\gamma(\tilde{f})-\gamma(f)) b t}\right) \rightarrow 0, \quad \text { as } t \rightarrow \infty
\end{aligned}
$$

(2) If $\alpha=2 \gamma(\tilde{f}) b$, then $\lim _{t \rightarrow \infty} t^{-1} e^{-\alpha t} \mathbb{P}_{\mu}\left\langle\tilde{f}, X_{t}\right\rangle^{2}$ exists. Thus we have

$$
\begin{aligned}
\mathbb{P}_{\mu} M_{t}^{2} & =t e^{-2(\gamma(\tilde{f})-\gamma(f)) t}\left(t^{-1} e^{-\alpha t} \mathbb{P}_{\mu}\left\langle\tilde{f}, X_{t}\right\rangle^{2}\right) \\
& =O\left(t e^{-2(\gamma(\tilde{f})-\gamma(f)) t}\right) \rightarrow 0, \quad \text { as } t \rightarrow \infty
\end{aligned}
$$

(3) If $2 \gamma(f) b<\alpha<2 \gamma(\tilde{f}) b$, then $\lim _{t \rightarrow \infty} e^{-\alpha t} \mathbb{P}_{\mu}\left\langle\tilde{f}, X_{t}\right\rangle^{2}$ exists. Thus we have

$$
\begin{aligned}
\mathbb{P}_{\mu} M_{t}^{2} & =e^{-(\alpha-2 \gamma(f) b) t}\left(e^{-\alpha t} \mathbb{P}_{\mu}\left\langle\tilde{f}, X_{t}\right\rangle^{2}\right) \\
& =O\left(e^{-(\alpha-2 \gamma(f) b) t}\right) \rightarrow 0, \quad \text { as } t \rightarrow \infty .
\end{aligned}
$$

Combining the three cases above, we get $\lim _{t \rightarrow \infty} M_{t}=0$ in $L^{2}\left(\mathbb{P}_{\mu}\right)$. Now using Lemma 3.1, we easily get the convergence in Theorem 1.1 .

\subsection{The Small Rate Case: $\alpha<2 \gamma(f) b$}

First, we recall some property of weak convergence. For $f: \mathbb{R}^{d} \rightarrow \mathbb{R}$, let $\|f\|_{L}:=$ $\sup _{x \neq y}|f(x)-f(y)| /\|x-y\|$ and $\|f\|_{B L}:=\|f\|_{\infty}+\|f\|_{L}$. For any distributions $v_{1}$ and $v_{2}$ on $\mathbb{R}^{d}$, define

$$
\beta\left(v_{1}, v_{2}\right):=\sup \left\{\left|\int f d v_{1}-\int f d v_{2}\right|:\|f\|_{B L} \leq 1\right\} .
$$

Then $\beta$ is a metric. By [12, Theorem 11.3.3], the topology generated by this metric is equivalent to the weak convergence topology. From the definition, we can easily see that, if $v_{1}$ and $\nu_{2}$ are the distributions of two $\mathbb{R}^{d}$-valued random variables $X$ and $Y$ respectively, then

$$
\beta\left(v_{1}, v_{2}\right) \leq E\|X-Y\| \leq \sqrt{E\|X-Y\|^{2}} .
$$

We will use the following elementary fact later: If $\mathrm{X}$ is a real-valued random variable with $E|X|^{n}<\infty$, then

$$
\left|E\left(e^{i \theta X}-\sum_{m=0}^{n} \frac{(i \theta X)^{m}}{m !}\right)\right| \leq \frac{|\theta|^{n}}{n !} E\left(|X|^{n}\left(\frac{|\theta X|}{n+1} \wedge 2\right)\right),
$$

which is an immediate consequence of the simple inequality

$$
\left|e^{i x}-\sum_{m=0}^{n} \frac{(i x)^{m}}{m !}\right| \leq \min \left(\frac{|x|^{n+1}}{(n+1) !}, \frac{2|x|^{n}}{n !}\right) .
$$

Now we are ready to prove Theorem 1.4.

Proof of Theorem 1.4 We define an $\mathbb{R}^{2}$-valued random variable $U_{1}(t)$ by

$$
U_{1}(t):=\left(e^{-\alpha t}\left\|\Lambda_{t}\right\|, e^{-(\alpha / 2) t}\left\langle f, \Lambda_{t}\right\rangle\right)
$$


To get the conclusion of Theorem 1.4, it suffices to show that, under $\mathbf{P}_{\mu}$,

$$
U_{1}(t) \stackrel{d}{\rightarrow}\left(W_{\infty}, \sqrt{W_{\infty}} G_{1}(f)\right),
$$

where $G_{1}(f) \sim \mathcal{N}\left(0, \sigma_{f}^{2}\right)$. Let $s, t>0$ and write

$$
U_{1}(s+t)=\left(e^{-\alpha(s+t)}\left\|\Lambda_{s+t}\right\|, e^{-(\alpha / 2)(s+t)}\left\langle f, \Lambda_{s+t}\right\rangle\right) .
$$

Recall the representation (3.1). Define

$$
Y_{s}^{u, t}:=e^{-\alpha s / 2}\left\langle f, I_{s}^{u, t}\right\rangle \quad \text { and } \quad y_{s}^{u, t}:=\mathbf{P}_{\mu}\left(Y_{s}^{u, t} \mid \mathcal{G}_{t}\right),
$$

here $\mathcal{G}_{t}$ is defined by (2.6). Given $\mathcal{G}_{t}, Y_{s}^{u, t}$ has the same law as $Y_{s}:=e^{-\alpha s / 2}\left\langle f, I_{s}\right\rangle$ under $\mathbb{Q}_{\delta_{z_{u}(t)}}$. So by (2.48),

$$
y_{s}^{u, t}=\frac{1}{\lambda^{*}} e^{-\alpha s / 2}\left(e^{\alpha s}-e^{-\alpha^{*} s}\right) T_{s} f\left(z_{u}(t)\right) .
$$

Then we have

$$
\begin{aligned}
e^{-(\alpha / 2)(s+t)}\left\langle f, \Lambda_{s+t}\right\rangle & \\
= & e^{-(\alpha / 2)(s+t)}\left\langle f, \tilde{X}_{s}^{t}\right\rangle+e^{-(\alpha / 2) t} \sum_{u \in \mathcal{L}_{t}} Y_{s}^{u, t} \\
= & e^{-(\alpha / 2)(s+t)}\left(\left\langle f, \tilde{X}_{s}^{t}\right\rangle-\mathbf{P}_{\mu}\left(\left\langle f, \tilde{X}_{s}^{t}\right\rangle \mid \mathcal{G}_{t}\right)\right)+e^{-(\alpha / 2) t} \sum_{u \in \mathcal{L}_{t}}\left(Y_{s}^{u, t}-y_{s}^{u, t}\right) \\
& +\left[e^{-(\alpha / 2)(s+t)} e^{-\alpha^{*} s}\left\langle T_{s} f, \Lambda_{t}\right\rangle+\frac{1}{\lambda^{*}} e^{-(\alpha / 2)(t+s)}\left(e^{\alpha s}-e^{-\alpha^{*} s}\right)\left\langle T_{s} f, Z_{t}\right\rangle\right] \\
= & : J_{0}(s, t)+J_{1}(s, t)+J_{2}(s, t) .
\end{aligned}
$$

Put $\widetilde{V}_{s}(x):=\operatorname{Var}_{\delta_{x}}\left\langle f, \widetilde{X}_{s}\right\rangle$. Then

$$
\mathbf{P}_{\mu} J_{0}(s, t)^{2}=e^{-\alpha(t+s)} \mathbf{P}_{\mu}\left\langle\widetilde{V}_{s}, \Lambda_{t}\right\rangle=e^{-\alpha s}\left\langle T_{t} \widetilde{V}_{s}, \mu\right\rangle .
$$

By (2.59), there exists $r \in \mathbb{N}$ such that $\widetilde{V}_{s}(x) \lesssim e^{-\alpha^{*} s}\left(1+\|x\|^{2 r}\right)$. Thus

$$
\mathbf{P}_{\mu} J_{0}(s, t)^{2} \lesssim e^{-\alpha s} e^{-\alpha^{*} s} \int_{\mathbb{R}^{d}}\left(1+\|x\|^{2 r}\right) \mu(d x)
$$

Next we consider $J_{2}(s, t)$. We have

$$
\mathbf{P}_{\mu} J_{2}(s, t)^{2} \leq 2 e^{-\alpha(s+t)} \mathbf{P}_{\mu}\left\langle T_{s} f, \Lambda_{t}\right\rangle^{2}+2 \frac{1}{\left(\lambda^{*}\right)^{2}} e^{\alpha s} e^{-\alpha t} \mathbf{P}_{\mu}\left\langle T_{s} f, Z_{t}\right\rangle^{2} .
$$

By (2.10) and (2.11), we have

$$
\begin{aligned}
e^{-\alpha t} \mathbf{P}_{\mu}\left\langle T_{s} f, \Lambda_{t}\right\rangle^{2} & =A \int_{\mathbb{R}^{d}} \int_{0}^{t} e^{\alpha u} T_{t-u}\left[T_{s+u} f\right]^{2}(x) d u \mu(d x)+e^{\alpha t}\left\langle T_{t+s} f, \mu\right\rangle^{2} \\
& \lesssim e^{-2 \gamma(f) b s} \int_{\mathbb{R}^{d}}\left(1+\|x\|^{2 r}\right) \mu(d x)
\end{aligned}
$$


here the last inequality follows from the fact that there exists $r \in \mathbb{N}$ such that

$$
\left|T_{s+u} f\right|(x) \lesssim e^{-\gamma(f) b(u+s)}\left(1+\|x\|^{r}\right)
$$

Since, given $\Lambda_{t}, Z_{t}$ is a Poisson random measure with intensity $\lambda^{*} \Lambda_{t}$, we have

$$
\begin{aligned}
e^{-\alpha t} \mathbf{P}_{\mu}\left\langle T_{s} f, Z_{t}\right\rangle^{2} & =\lambda^{*} e^{-\alpha t} \mathbf{P}_{\mu}\left\langle\left(T_{s} f\right)^{2}, \Lambda_{t}\right\rangle+\left(\lambda^{*}\right)^{2} e^{-\alpha t} \mathbf{P}_{\mu}\left\langle T_{s} f, \Lambda_{t}\right\rangle^{2} \\
& =\lambda^{*}\left\langle T_{t}\left(T_{s} f\right)^{2}, \mu\right\rangle+\left(\lambda^{*}\right)^{2} e^{-\alpha t} \mathbf{P}_{\mu}\left\langle T_{s} f, \Lambda_{t}\right\rangle^{2} \\
& \lesssim e^{-2 \gamma(f) b s} \int_{\mathbb{R}^{d}}\left(1+\|x\|^{2 r}\right) \mu(d x),
\end{aligned}
$$

here the last inequality follows from (3.12) and (3.13). Thus by (3.11), (3.12) and (3.14), we get

$$
\mathbf{P}_{\mu} J_{2}(s, t)^{2} \lesssim e^{(\alpha-2 \gamma(f) b) s} \int_{\mathbb{R}^{d}}\left(1+\|x\|^{2 r}\right) \mu(d x) .
$$

Thus by (3.10) and (3.15), we have

$$
\lim _{s \rightarrow \infty} \limsup _{t \rightarrow \infty} \mathbf{P}_{\mu}\left(J_{0}(s, t)+J_{2}(s, t)\right)^{2}=0 .
$$

Now we consider $J_{1}(s, t)$. We define an $\mathbb{R}^{2}$-valued random variable $U_{2}(s, t)$ by

$$
U_{2}(s, t):=\left(e^{-\alpha t}\left\|\Lambda_{t}\right\|, J_{1}(s, t)\right)
$$

We claim that, under $\mathbf{P}_{\mu}$,

$$
U_{2}(s, t) \stackrel{d}{\rightarrow}\left(W_{\infty}, \sqrt{W_{\infty}} G_{1}(s)\right), \quad \text { as } t \rightarrow \infty,
$$

where $G_{1}(s) \sim \mathcal{N}\left(0, \sigma_{f}^{2}(s)\right)$ and $\sigma_{f}^{2}(s)$ will be given later. Denote the characteristic function of $U_{2}(s, t)$ under $\mathbf{P}_{\mu}$ by $\kappa\left(\theta_{1}, \theta_{2}, s, t\right)$ :

$$
\begin{aligned}
\kappa\left(\theta_{1}, \theta_{2}, s, t\right) & =\mathbf{P}_{\mu}\left(\exp \left\{i \theta_{1} e^{-\alpha t}\left\|\Lambda_{t}\right\|+i \theta_{2} e^{-(\alpha / 2) t} \sum_{u \in \mathcal{L}_{t}}\left(Y_{s}^{u, t}-y_{s}^{u, t}\right)\right\}\right) \\
& =\mathbf{P}_{\mu}\left(\exp \left\{i \theta_{1} e^{-\alpha t}\left\|\Lambda_{t}\right\|\right\} \prod_{u \in \mathcal{L}_{t}} h_{s}\left(z_{u}(t), e^{-(\alpha / 2) t} \theta_{2}\right)\right) \\
& =\mathbf{P}_{\mu}\left(\exp \left\{i \theta_{1} e^{-\alpha t}\left\|\Lambda_{t}\right\|\right\} \exp \left\{\lambda^{*}\left\{h_{s}\left(\cdot, e^{-(\alpha / 2) t} \theta_{2}\right)-1, \Lambda_{t}\right\rangle\right\}\right),
\end{aligned}
$$

where $h_{s}(x, \theta)=\mathbb{Q}_{\delta_{x}} e^{i \theta\left(Y_{s}-\mathbb{Q}_{\delta_{x}} Y_{s}\right)}$. The last equality in the display above follows from the fact that, given $\Lambda_{t}, Z_{t}$ is a Poisson random measure with intensity $\lambda^{*} \Lambda_{t}$. Define

$$
e_{s}(x, \theta):=h_{s}(x, \theta)-1+\frac{1}{2} \theta^{2} \mathbb{V}_{\delta_{x}} Y_{s}
$$

and $V_{s}(x):=\mathbb{V}_{\delta_{x}} Y_{s}$. Then

$$
\begin{aligned}
\exp \left\{\lambda^{*}\left\langle h_{s}\left(\cdot, e^{-(\alpha / 2) t} \theta_{2}\right)-1, \Lambda_{t}\right\rangle\right\} & =\exp \left\{-\lambda^{*} \frac{1}{2} \theta_{2}^{2} e^{-\alpha t}\left\langle V_{s}, \Lambda_{t}\right\rangle\right\} \exp \left\{\lambda^{*}\left\langle e_{s}\left(\cdot, e^{-(\alpha / 2) t} \theta_{2}\right), \Lambda_{t}\right\rangle\right\} \\
& =J_{1,1}(s, t) J_{1,2}(s, t) .
\end{aligned}
$$


By (3.5), we have

$$
\left|e_{s}\left(x, e^{-(\alpha / 2) t} \theta_{2}\right)\right| \leq \theta_{2}^{2} e^{-\alpha t} \mathbb{Q}_{\delta_{x}}\left(\left|Y_{s}-\mathbb{Q}_{\delta_{x}} Y_{s}\right|^{2}\left(\frac{e^{-(\alpha / 2) t} \theta_{2}\left|Y_{s}-\mathbb{Q}_{\delta_{x}} Y_{s}\right|}{6} \wedge 1\right)\right) .
$$

Let

$$
g(x, s, t):=\mathbb{Q}_{\delta_{x}}\left(\left|Y_{s}-\mathbb{Q}_{\delta_{x}} Y_{s}\right|^{2}\left(\frac{e^{-(\alpha / 2) t} \theta_{2}\left|Y_{s}-\mathbb{Q}_{\delta_{x}} Y_{s}\right|}{6} \wedge 1\right)\right) .
$$

By (2.10),

$$
\mathbf{P}_{\mu}\left|\left\langle e_{s}\left(\cdot, e^{-(\alpha / 2) t} \theta_{2}\right), \Lambda_{t}\right\rangle\right| \leq \theta_{2}^{2}\left\langle T_{t}(g(\cdot, s, t)), \mu\right\rangle .
$$

We notice that $g(x, s, t) \downarrow 0$ as $t \uparrow \infty$. For any $u<t$,

$$
\limsup _{t \rightarrow \infty} T_{t}(g(\cdot, s, t)) \leq \limsup _{t \rightarrow \infty} T_{t}(g(\cdot, s, u))=\langle g(\cdot, s, u), \varphi\rangle .
$$

Then letting $u \rightarrow \infty$, we get $\lim _{t \rightarrow \infty}\left\langle e_{s}\left(\cdot, e^{-(\alpha / 2) t} \theta_{2}\right), \Lambda_{t}\right\rangle=0$ in probability, which implies that $\lim _{t \rightarrow \infty} J_{1,2}(s, t)=1$ in probability. Furthermore, by Remark 1.2, we have

$$
\lim _{t \rightarrow \infty} e^{-\alpha t}\left\langle V_{s}, \Lambda_{t}\right\rangle=\left\langle V_{s}, \varphi\right\rangle W_{\infty} \quad \text { in probability, }
$$

which implies that $\lim _{t \rightarrow \infty} J_{1,1}(s, t)=\exp \left\{-\frac{1}{2} \theta_{2}^{2} \sigma_{f}^{2}(s) W_{\infty}\right\}$, where $\sigma_{f}^{2}(s):=\lambda^{*}\left\langle V_{s}, \varphi\right\rangle$. Thus

$$
\lim _{t \rightarrow \infty} \exp \left\{\lambda^{*}\left\langle h_{s}\left(\cdot, e^{-(\alpha / 2) t} \theta_{2}\right)-1, \Lambda_{t}\right\rangle\right\}=\exp \left\{-\frac{1}{2} \theta_{2}^{2} \sigma_{f}^{2}(s) W_{\infty}\right\} \quad \text { in probability. }
$$

Since $h_{s}(x, \theta)$ is a characteristic function, its real part is less than 1 , which implies

$$
\left|\exp \left\{\lambda^{*}\left\langle h_{s}\left(\cdot, e^{-(\alpha / 2) t} \theta_{2}\right)-1, \Lambda_{t}\right\rangle\right\}\right| \leq 1 .
$$

Hence by the dominated convergence theorem, we get

$$
\lim _{t \rightarrow \infty} \kappa\left(\theta_{1}, \theta_{2}, s, t\right)=\mathbf{P}_{\mu} \exp \left\{i \theta_{1} W_{\infty}\right\} \exp \left\{-\frac{1}{2} \theta_{2}^{2} \sigma_{f}^{2}(s) W_{\infty}\right\},
$$

which implies our claim (3.17). Since $e^{-\alpha(t+s)}\left\|\Lambda_{t+s}\right\|-e^{-\alpha t}\left\|\Lambda_{t}\right\| \rightarrow 0$ in probability as $t \rightarrow \infty$, we easily get that, under $\mathbf{P}_{\mu}$,

$$
U_{3}(s, t):=\left(e^{-\alpha(t+s)}\left\|\Lambda_{t+s}\right\|, J_{1}(s, t)\right) \stackrel{d}{\rightarrow}\left(W_{\infty}, \sqrt{W_{\infty}} G_{1}(s)\right), \quad \text { as } t \rightarrow \infty .
$$

By (2.51), we have $\lim _{s \rightarrow \infty} V_{s}(x)=\frac{\sigma_{f}^{2}}{\lambda^{*}}$, thus $\lim _{s \rightarrow \infty} \sigma_{f}^{2}(s)=\sigma_{f}^{2}$. So

$$
\lim _{s \rightarrow \infty} \beta\left(G_{1}(s), G_{1}(f)\right)=0 .
$$

Let $\mathcal{D}(s+t)$ and $\widetilde{\mathcal{D}}(s, t)$ be the distributions of $U_{1}(s+t)$ and $U_{3}(s, t)$ respectively, and let $\mathcal{D}(s)$ and $\mathcal{D}$ be the distributions of $\left(W_{\infty}, \sqrt{W_{\infty}} G_{1}(s)\right)$ and $\left(W_{\infty}, \sqrt{W_{\infty}} G_{1}(f)\right)$ respectively. Then, using (3.4), we have

$$
\limsup _{t \rightarrow \infty} \beta(\mathcal{D}(s+t), \mathcal{D}) \leq \limsup _{t \rightarrow \infty}[\beta(\mathcal{D}(s+t), \widetilde{\mathcal{D}}(s, t))+\beta(\widetilde{\mathcal{D}}(s, t), \mathcal{D}(s))+\beta(\mathcal{D}(s), \mathcal{D})]
$$




$$
\leq \limsup _{t \rightarrow \infty}\left(\mathbf{P}_{\mu}\left(J_{0}(s, t)+J_{2}(s, t)\right)^{2}\right)^{1 / 2}+0+\beta(\mathcal{D}(s), \mathcal{D}) .
$$

Using this and the definition of $\limsup _{t \rightarrow \infty}$, we easily get that

$$
\begin{aligned}
\limsup _{t \rightarrow \infty} \beta(\mathcal{D}(t), \mathcal{D}) & =\limsup _{t \rightarrow \infty} \beta(\mathcal{D}(s+t), \mathcal{D}) \\
& \leq \limsup _{t \rightarrow \infty}\left(\mathbf{P}_{\mu}\left(J_{0}(s, t)+J_{2}(s, t)\right)^{2}\right)^{1 / 2}+\beta(\mathcal{D}(s), \mathcal{D}) .
\end{aligned}
$$

Letting $s \rightarrow \infty$, we get $\lim \sup _{t \rightarrow \infty} \beta(\mathcal{D}(t), \mathcal{D})=0$. The proof is now complete.

\subsection{Proof of Theorem 1.10}

In this section we consider the case $\alpha>2 \gamma(f) b$ and $f_{(c)}=0$. Recalling the decomposition of $\Lambda_{t}$ under $\mathbf{P}_{\delta_{x}}$ in (2.46), we have for $|p|=m<\alpha /(2 b)$,

$$
H_{s}^{p}=e^{-(\alpha-m b) s}\left\langle\phi_{p}, \tilde{X}_{s}\right\rangle+\sum_{j=1}^{N} e^{-(\alpha-m b) s}\left\langle\phi_{p}, I_{s}^{j}\right\rangle .
$$

Let

$$
\widetilde{H}_{s}^{p}:=e^{-(\alpha-m b) s}\left\langle\phi_{p}, I_{s}\right\rangle
$$

Then, under $\mathbf{P}_{\delta_{x}}$, the processes $\left\{e^{-(\alpha-m b) s}\left\langle\phi_{p}, I_{s}^{j}\right\rangle, s \geq 0\right\}, j=1,2 \ldots$ are i.i.d. with a common law equal to that of $\left\{\widetilde{H}_{s}^{p}, s \geq 0\right\}$ under $\mathbb{Q}_{\delta_{x}}$. Since $\phi_{p}$ is an eigenvalue of $L$ corresponding to $-|p| b$, we have

$$
\mathbf{P}_{\delta_{x}}\left\langle\phi_{p}, \tilde{X}_{s}\right\rangle=e^{-\left(\alpha^{*}+m b\right) s} \phi_{p}(x) \rightarrow 0, \quad \text { as } s \rightarrow \infty .
$$

Thus, by (2.59), we have that as $s \rightarrow \infty$,

$$
\mathbf{P}_{\delta_{x}}\left(\left\langle\phi_{p}, \tilde{X}_{s}\right\rangle\right)^{2} \lesssim e^{-\alpha^{*} s}\left(1+\|x\|^{2|p|}\right) \rightarrow 0
$$

which implies $\lim _{s \rightarrow \infty} e^{-(\alpha-m b) s}\left\langle\phi_{p}, \tilde{X}_{s}\right\rangle=0$ in $L^{2}\left(\mathbf{P}_{\delta_{x}}\right)$. By Lemma 3.1, $\lim _{s \rightarrow \infty} H_{s}^{p}=H_{\infty}^{p}$ in $L^{2}\left(\mathbf{P}_{\delta_{x}}\right)$. Thus

$$
\lim _{s \rightarrow \infty} \sum_{j=1}^{N} e^{-(\alpha-m b) s}\left\langle\phi_{p}, I_{s}^{j}\right\rangle=H_{\infty}^{p} \quad \text { in } L^{2}\left(\mathbf{P}_{\delta_{x}}\right) .
$$

From the fact that $N$ is independent of $I^{j}$, we have for any $s, t \geq 0$,

$$
\begin{aligned}
\mathbf{P}_{\delta_{x}} & {\left[\sum_{j=1}^{N}\left(e^{-(\alpha-m b) s}\left\langle\phi_{p}, I_{s}^{j}\right\rangle-e^{-(\alpha-m b) t}\left\langle\phi_{p}, I_{t}^{j}\right\rangle\right)\right]^{2} } \\
\geq & \mathbf{P}_{\delta_{x}}\left[\left(e^{-(\alpha-m b) s}\left\langle\phi_{p}, I_{s}\right\rangle-e^{-(\alpha-m b) t}\left\langle\phi_{p}, I_{t}\right\rangle\right)^{2} ; N=1\right] \\
& =\mathbf{P}_{\delta_{x}}(N=1) \mathbb{Q}_{\delta_{x}}\left(\widetilde{H}_{s}^{p}-\widetilde{H}_{t}^{p}\right)^{2} .
\end{aligned}
$$

By (3.26), we get for any $x \in \mathbb{R}^{d}$,

$$
\mathbb{Q}_{\delta_{x}}\left(\widetilde{H}_{s}^{p}-\widetilde{H}_{t}^{p}\right)^{2} \rightarrow 0, \quad s, t \rightarrow \infty .
$$


Thus $\widetilde{H}_{s}^{p}$ converges in $L^{2}\left(\mathbb{Q}_{\delta_{x}}\right)$. Let

$$
\widetilde{H}_{\infty}^{p}:=\lim _{s \rightarrow \infty} \widetilde{H}_{s}^{p} \quad \text { in } L^{2}\left(\mathbb{Q}_{\delta_{x}}\right),
$$

which implies $H_{\infty}^{j, p}:=\lim _{s \rightarrow \infty}\left\langle\phi_{p}, I_{s}^{j}\right\rangle e^{-(\alpha-m b) s}$ exists in $L^{2}\left(\mathbf{P}_{\delta_{x}}\right)$. Furthermore, $H_{\infty}^{j, p}$, under $\mathbf{P}_{\delta_{x}}$, are i.i.d. with a common law equal to that of $\widetilde{H}_{\infty}^{p}$ under $\mathbb{Q}_{\delta_{x}}$. Hence by (3.26), it is easy to get

$$
H_{\infty}^{p}=\sum_{j=1}^{N} H_{\infty}^{j, p}, \quad \mathbf{P}_{\delta_{x}} \text {-a.s. }
$$

Recall the decomposition of $\Lambda_{t+s}$ in (3.1). By Lemma 3.1, we have for $|p|=m<$ $\alpha /(2 b)$,

$$
H_{t+s}^{p}=e^{-(\alpha-m b)(s+t)}\left\langle\phi_{p}, \tilde{X}_{s}^{t}\right\rangle+e^{-(\alpha-m b) t} \sum_{u \in \mathcal{L}_{t}} e^{-(\alpha-m b) s}\left\langle\phi_{p}, I_{s}^{u, t}\right\rangle .
$$

From the definition of $\tilde{X}_{s}^{t}$, using (2.59) and (3.24), we have

$$
\begin{aligned}
\mathbf{P}_{\mu}\left(\left\langle\phi_{p}, \tilde{X}_{s}^{t}\right\rangle\right)^{2} & \leq 2 \mathbf{P}_{\mu}\left(\left\langle\phi_{p}, \tilde{X}_{s}^{t}\right\rangle-\mathbf{P}_{\mu}\left(\left|\phi_{p}, \tilde{X}_{s}^{t}\right\rangle \mid \mathcal{F}_{t}\right)\right)^{2}+2 \mathbf{P}_{\mu}\left(\mathbf{P}_{\mu}\left(\left\langle\phi_{p}, \tilde{X}_{s}^{t}\right\rangle \mid \mathcal{F}_{t}\right)\right)^{2} \\
& =2 \mathbf{P}_{\mu}\left\langle\mathbf{V} \operatorname{rar}_{\delta}\left\langle\phi_{p}, \widetilde{X}_{s}\right\rangle, \Lambda_{t}\right\rangle+2 \mathbf{P}_{\mu}\left\langle\mathbf{P}_{\delta .}\left\langle\phi_{p}, \widetilde{X}_{s}\right\rangle, \Lambda_{t}\right\rangle^{2} \rightarrow 0, \quad \text { as } s \rightarrow \infty .
\end{aligned}
$$

Hence $\lim _{s \rightarrow \infty} e^{-(\alpha-m b)(s+t)}\left\langle\phi_{p}, \tilde{X}_{s}^{t}\right\rangle=0$ in $L^{2}\left(\mathbf{P}_{\mu}\right)$. Thus $\lim _{s \rightarrow \infty} e^{-(\alpha-m b)(s+t)}\left\langle\phi_{p}, \widetilde{X}_{s}^{t}\right\rangle=0$ in $L^{2}\left(\mathbf{P}_{\mu}\right)$. Thus

$$
\lim _{s \rightarrow \infty} e^{-(\alpha-m b) t} \sum_{u \in \mathcal{L}_{t}}\left\langle\phi_{p}, I_{s}^{u, t}\right\rangle e^{-(\alpha-m b) s}=H_{\infty}^{p} \quad \text { in } L^{2}\left(\mathbf{P}_{\mu}\right)
$$

Note that under $\mathbf{P}_{\mu}$, given $Z_{t}, e^{-(\alpha-m b) s}\left\langle\phi_{p}, I_{s}^{u, t}\right\rangle$ has the same law as $\widetilde{H}_{s}^{p}$ under $\mathbb{Q}_{\delta_{z_{u}(t)}}$. Thus by (3.27), for each $u \in \mathcal{L}_{t}, e^{-(\alpha-m b) s}\left\langle\phi_{p}, I_{s}^{u, t}\right\rangle$ converges in $L^{2}\left(\mathbf{P}_{\mu}\right)$ to a limit, denoted as $H_{\infty}^{u, t, p}$. Furthermore, given $Z_{t}, H_{\infty}^{u, t, p}$ has the same law as $\widetilde{H}_{\infty}^{p}$ under $\mathbb{Q}_{\delta_{z_{u}(t)}}$.

We claim that, for each $t \geq 0$,

$$
H_{\infty}^{p}=e^{-(\alpha-m b) t} \sum_{u \in \mathcal{L}_{t}} H_{\infty}^{u, t, p} .
$$

In fact,

$$
\begin{aligned}
\mathbf{P}_{\mu}\left(\sum_{u \in \mathcal{L}_{t}} e^{-(\alpha-m b) s}\left\langle\phi_{p}, I_{s}^{u, t}\right\rangle-H_{\infty}^{u, t, p}\right)^{2} & \leq \mathbf{P}_{\mu}\left|Z_{t}\right| \sum_{u \in \mathcal{L}_{t}}\left(e^{-(\alpha-m b) s}\left\langle\phi_{p}, I_{s}^{u, t}\right\rangle-H_{\infty}^{u, t, p}\right)^{2} \\
& =\mathbf{P}_{\mu}\left|Z_{t}\right| \sum_{u \in \mathcal{L}_{t}} \mathbb{Q}_{\delta_{Z_{u}(t)}}\left(\widetilde{H}_{s}^{p}-\widetilde{H}_{\infty}^{p}\right)^{2}
\end{aligned}
$$

By (2.49), we have

$$
\mathbb{Q}_{\delta_{x}}\left(\widetilde{H}_{s}^{p}\right)^{2} \leq \frac{1}{\lambda^{*}} \operatorname{Var}_{\delta_{x}}\left(H_{s}^{p}\right) \leq \frac{1}{\lambda^{*}} \mathbb{P}_{\delta_{x}}\left(H_{s}^{p}\right)^{2} \lesssim 1+\|x\|^{2|p|} .
$$


Thus $\mathbb{Q}_{\delta_{x}}\left(\widetilde{H}_{s}^{p}-\widetilde{H}_{\infty}^{p}\right)^{2} \leq 2 \sup _{s \geq 0} \mathbb{Q}_{\delta_{x}}\left(\widetilde{H}_{s}^{p}\right)^{2} \lesssim 1+\|x\|^{2|p|}$. We can easily get that

$$
\mathbf{P}_{\mu}\left|Z_{t}\right|\left\langle\left(1+\|\cdot\|^{2|p|}\right), Z_{t}\right\rangle<\infty .
$$

So by the dominated convergence theorem, we have $\lim _{s \rightarrow \infty} \mathbf{P}_{\mu}\left(\sum_{u \in \mathcal{L}_{t}} e^{-(\alpha-m b) s}\left\langle\phi_{p}, I_{s}^{u, t}\right\rangle-\right.$ $\left.H_{\infty}^{u, t, p}\right)^{2}=0$. Now the claim (3.31) follows easily from (3.30).

Define

$$
H_{\infty}^{u, t}:=\sum_{\gamma(f) \leq m<\alpha / 2 b} \sum_{|p|=m} a_{p} H_{\infty}^{u, t, p} \text { and } \quad \widetilde{H}_{\infty}:=\sum_{\gamma(f) \leq m<\alpha / 2 b|p|=m} \sum_{p} a_{p} \widetilde{H}_{\infty}^{p} .
$$

Recall the definition of $H_{\infty}$ in (1.19). By (3.28), we have

$$
H_{\infty}=\sum_{u \in \mathcal{L}_{0}} H_{\infty}^{u, 0}
$$

Under $\mathbf{P}_{\delta_{x}}, H_{\infty}^{u, 0}$ are i.i.d. with a common law equal to that of $\widetilde{H}_{\infty}$ under $\mathbb{Q}_{\delta_{x}}$. Thus we have

$$
\begin{aligned}
\mathbf{P}_{\delta_{x}} H_{\infty} & =\lambda^{*} \mathbb{Q}_{\delta_{x}} \widetilde{H}_{\infty}, \\
\mathbf{V}^{a r_{\delta_{x}}} H_{\infty} & =\lambda^{*} \mathbb{Q}_{\delta_{x}}\left(\widetilde{H}_{\infty}\right)^{2} .
\end{aligned}
$$

On the other hand, by Lemma 3.1, we get

$$
\lim _{t \rightarrow \infty} \sum_{\gamma(f) \leq m<\alpha / 2 b} e^{-(\alpha-m b) t} \sum_{|p|=m} a_{p}\left\langle\phi_{p}, \Lambda_{t}\right\rangle=H_{\infty}, \quad \text { in } L^{2}\left(\mathbf{P}_{\delta_{x}}\right) .
$$

It follows that

$$
\mathbf{P}_{\delta_{x}} H_{\infty}=f_{(s)}(x)
$$

and by (2.41),

$$
\mathbf{V} a r_{\delta_{x}} H_{\infty}=A \int_{0}^{\infty} e^{-\alpha s} T_{s}\left(\sum_{\gamma(f) \leq m<\alpha / 2 b} e^{m b s} \sum_{|p|=m} a_{p} \phi_{p}\right)^{2}(x) d s .
$$

Proof of Theorem 1.10 By (3.31), we have

$$
\sum_{\gamma(f) \leq m<\alpha / 2 b} e^{(\alpha-m b) t} \sum_{|p|=m} a_{p} H_{\infty}^{p}=\sum_{u \in \mathcal{L}_{t}} H_{\infty}^{u, t} .
$$

Consider the $\mathbb{R}^{2}$-valued random variable $U_{1}(t)$ :

$$
U_{1}(t):=\left(e^{-\alpha t}\left\|\Lambda_{t}\right\|, e^{-(\alpha / 2) t}\left(\left\langle f, \Lambda_{t}\right\rangle-\sum_{u \in \mathcal{L}_{t}} H_{\infty}^{u, t}\right)\right) .
$$

To get the conclusion of Theorem 1.10, it suffices to show that

$$
U_{1}(t) \stackrel{d}{\rightarrow}\left(W_{\infty}, \sqrt{W_{\infty}} G_{3}(f)\right) .
$$


Denote the characteristic function of $U_{1}(t)$ under $\mathbf{P}_{\mu}$ by $\kappa_{1}\left(\theta_{1}, \theta_{2}, t\right)$ and let $h(x, \theta):=$ $\mathbb{Q}_{\delta_{x}} \exp \left\{i \theta \widetilde{H}_{\infty}\right\}$. Then we have

$$
\begin{aligned}
& \kappa_{1}\left(\theta_{1}, \theta_{2}, t\right) \\
& =\mathbf{P}_{\mu} \exp \left\{i \theta_{1} e^{-\alpha t}\left\|\Lambda_{t}\right\|+i \theta_{2} e^{-(\alpha / 2) t}\left(\left\langle f, \Lambda_{t}\right\rangle-\sum_{u \in \mathcal{L}_{t}} H_{\infty}^{u, t}\right)\right\} \\
& =\mathbf{P}_{\mu} \exp \left\{i \theta_{1} e^{-\alpha t}\left\|\Lambda_{t}\right\|\right\} \exp \left\{i \theta_{2} e^{-(\alpha / 2) t}\left\langle f, \Lambda_{t}\right\rangle\right\} \prod_{u \in \mathcal{L}_{t}} h\left(Z_{u}(t),-\theta_{2} e^{-(\alpha / 2) t}\right) \\
& =\mathbf{P}_{\mu} \exp \left\{i \theta_{1} e^{-\alpha t}\left\|\Lambda_{t}\right\|\right\} \exp \left\{i \theta_{2} e^{-(\alpha / 2) t}\left\langle f, \Lambda_{t}\right\rangle+\lambda^{*}\left\{h\left(\cdot,-\theta_{2} e^{-(\alpha / 2) t}\right)-1, \Lambda_{t}\right\rangle\right\} .
\end{aligned}
$$

The third equality above follows from the fact that, given $\Lambda_{t}, Z_{t}$ is a Poisson point process with density $\lambda^{*} \Lambda_{t}$. By (3.32) and (3.34), we get $\mathbb{Q}_{\delta_{x}} \widetilde{H}_{\infty}=f_{(s)}(x) / \lambda^{*}$. Let

$$
e(x, \theta):=h(x, \theta)-1-\frac{i \theta}{\lambda^{*}} f_{(s)}(x)+\frac{1}{2} \mathbb{Q}_{\delta_{x}}\left(\widetilde{H}_{\infty}\right)^{2} \theta^{2}
$$

and $V(x):=\mathbf{V}^{a} r_{\delta_{x}} H_{\infty}$. Then, by (3.33), we have

$$
\begin{aligned}
& i \theta_{2} e^{-(\alpha / 2) t}\left\langle f, \Lambda_{t}\right\rangle+\lambda^{*}\left\langle h\left(\cdot,-\theta_{2} e^{-(\alpha / 2) t}\right)-1, \Lambda_{t}\right\rangle \\
& \quad=i \theta_{2} e^{-(\alpha / 2) t}\left\langle f_{(l)}, \Lambda_{t}\right\rangle-\frac{1}{2} \theta_{2}^{2} e^{-\alpha t}\left\langle V, \Lambda_{t}\right\rangle+\lambda^{*}\left\langle e\left(\cdot,-\theta_{2} e^{-(\alpha / 2) t}\right), \Lambda_{t}\right\rangle \\
& \quad=: J_{1}(t)+J_{2}(t)+J_{3}(t) .
\end{aligned}
$$

By (3.5), we have

$$
|e(x, \theta)| \leq \theta^{2} \mathbb{Q}_{\delta_{x}}\left(\left|\widetilde{H}_{\infty}\right|^{2}\left(\frac{\theta\left|\widetilde{H}_{\infty}\right|}{6} \wedge 1\right)\right)
$$

which implies that

$$
\left|J_{3}(t)\right| \leq \theta_{2}^{2} e^{-\alpha t}\left\langle g(\cdot, t), \Lambda_{t}\right\rangle
$$

where

$$
g(x, t):=\mathbb{Q}_{\delta_{x}}\left(\left|\widetilde{H}_{\infty}\right|^{2}\left(\frac{e^{-(\alpha / 2) t} \theta_{2}\left|\tilde{H}_{\infty}\right|}{6} \wedge 1\right)\right) .
$$

It is clear that $g(x, t) \downarrow 0$ as $t \uparrow \infty$. Thus

$$
\mathbf{P}_{\mu}\left|J_{3}(t)\right| \leq \theta_{2}^{2}\left\langle T_{t}(g(\cdot, t)), \mu\right\rangle \rightarrow 0, \quad \text { as } t \rightarrow \infty,
$$

which implies $\lim _{t \rightarrow \infty} J_{3}(t)=0$ in probability. By Remark 1.2, we have

$$
\lim _{t \rightarrow \infty} e^{-\alpha t}\left\langle V, \Lambda_{t}\right\rangle=\langle V, \varphi\rangle W_{\infty} \quad \text { in probability. }
$$

Recall that $\lim _{t \rightarrow \infty} e^{-\alpha t}\left\|\Lambda_{t}\right\|=W_{\infty}, \mathbf{P}_{\mu}$-a.s. Therefore

$$
\begin{aligned}
& \lim _{t \rightarrow \infty} \exp \left\{i \theta_{1} e^{-\alpha t}\left\|\Lambda_{t}\right\|\right\} \exp \left\{J_{2}(t)+J_{3}(t)\right\}=\exp \left\{i \theta_{1} W_{\infty}\right\} \exp \left\{-\frac{1}{2} \theta_{2}^{2}\langle V, \varphi\rangle W_{\infty}\right\} \\
& \quad \text { in probability. }
\end{aligned}
$$


Thus by the dominated convergence theorem, we get that as $t \rightarrow \infty$,

$$
\left|\kappa_{1}\left(\theta_{1}, \theta_{2}, t\right)-\mathbf{P}_{\mu} \exp \left\{i \theta_{2} e^{-(\alpha / 2) t}\left\langle f_{(l)}, \Lambda_{t}\right\rangle\right\} \exp \left\{i \theta_{1} W_{\infty}\right\} \exp \left\{-\frac{1}{2} \theta_{2}^{2}\langle V, \varphi\rangle W_{\infty}\right\}\right| \rightarrow 0 .
$$

Since $\alpha<2 \gamma\left(f_{(l)}\right) b$, by Theorem 1.4, we have that as $t \rightarrow \infty$,

$$
\left(e^{-\alpha t}\left\|\Lambda_{t}\right\|, e^{-(\alpha / 2) t}\left\langle f_{(l)}, \Lambda_{t}\right\rangle\right) \stackrel{d}{\rightarrow}\left(W_{\infty}, \sqrt{W_{\infty}} G_{1}\left(f_{(l)}\right)\right),
$$

where $G_{1}\left(f_{(l)}\right) \sim \mathcal{N}\left(0, \sigma_{f_{(l)}}^{2}\right)$. Therefore,

$$
\begin{aligned}
& \lim _{t \rightarrow \infty} \mathbf{P}_{\mu} \exp \left\{i \theta_{2} e^{-(\alpha / 2) t}\left\langle f_{(l)}, \Lambda_{t}\right\rangle\right\} e^{i \theta_{1} W_{\infty}} \exp \left\{-\frac{1}{2} \theta_{2}^{2}\langle V, \varphi\rangle W_{\infty}\right\} \\
& \quad=\mathbf{P}_{\mu} e^{i \theta_{1} W_{\infty}} \exp \left\{-\frac{1}{2} \theta_{2}^{2}\left(\sigma_{f_{(l)}}^{2}+\langle V, \varphi\rangle\right) W_{\infty}\right\} .
\end{aligned}
$$

By (3.35), we get

$$
\langle V, \varphi\rangle=A \sum_{\gamma(f) \leq m<\alpha / 2 b} \frac{1}{\alpha-2 m b} \sum_{|p|=m} a_{p}^{2} .
$$

The proof is now complete.

3.4 The Critical Case: $\alpha=2 \gamma(f) b$

To prove Theorem 1.6, we need the following lemma. The idea of the proof is mainly from [8].

Lemma 3.2 Assume $f(x)=\sum_{|p|=k} b_{p} \phi_{p}(x)$, where $b_{p} \in \mathbb{R}$ and $\alpha=2 k b$. Define $T_{t}^{\alpha} f(x):=$ $e^{\alpha t} T_{t} f(x)=\mathbb{P}_{\delta_{x}}\left\langle f, X_{t}\right\rangle$ and

$$
S_{t} f(x):=t^{-1 / 2} e^{-(\alpha / 2) t}\left(\left\langle f, X_{t}\right\rangle-T_{t}^{\alpha} f(x)\right) .
$$

Then for any $c>0$ and $\delta>0$, we have

$$
\lim _{t \rightarrow \infty} \mathbb{P}_{\delta_{x}}\left(\left|S_{t} f(x)\right|^{2} ;\left|S_{t} f(x)\right|>c e^{\delta t}\right)=0 .
$$

Proof We write $t=[t]+\epsilon_{t}$, where $[t]$ is the integer part of $t$. Let

$$
F(t, x):=\mathbb{P}_{\delta_{x}}\left(\left|S_{t} f\right|^{2} ;\left|S_{t} f\right|>c e^{\delta t}\right) .
$$

By the definition of $f$, we get $T_{u}^{\alpha} f(x)=e^{\alpha u / 2} f(x)$. Note that

$$
\begin{aligned}
S_{t+1} f(x)= & \left(\frac{1}{t+1}\right)^{1 / 2} e^{-(\alpha / 2)(t+1)}\left(\left\langle f, X_{t+1}\right\rangle-\left\langle e^{\alpha / 2} f, X_{t}\right\rangle\right) \\
& +\left(\frac{1}{t+1}\right)^{1 / 2} e^{-(\alpha / 2) t}\left(\left\langle f, X_{t}\right\rangle-T_{t}^{\alpha} f(x)\right) \\
= & \left(\frac{1}{t+1}\right)^{1 / 2} R(t, f)+\left(\frac{t}{t+1}\right)^{1 / 2} S_{t} f(x),
\end{aligned}
$$


where $R(t, f):=e^{-(\alpha / 2)(t+1)}\left(\left\langle f, X_{t+1}\right\rangle-\left\langle T_{1}^{\alpha} f, X_{t}\right\rangle\right)$. Thus we have

$$
\begin{aligned}
F(t+1, x) \leq & \mathbb{P}_{\delta_{x}}\left(\left|S_{t+1} f(x)\right|^{2} ;\left|S_{t} f(x)\right|>c e^{\delta t}\right) \\
& +\mathbb{P}_{\delta_{x}}\left(\left|S_{t+1} f(x)\right|^{2} ;\left|S_{t} f(x)\right| \leq c e^{\delta t},\left|S_{t+1} f(x)\right|>c e^{\delta(t+1)}\right) \\
= & : M_{1}(t, x)+M_{2}(t, x) .
\end{aligned}
$$

Put

$$
\begin{aligned}
& A_{1}(t, x)=\left\{\left|S_{t} f(x)\right|>c e^{\delta t}\right\}, \\
& A_{2}(t, x)=\left\{\left|S_{t} f(x)\right| \leq c e^{\delta t},\left|S_{t+1} f(x)\right|>c e^{\delta(t+1)}\right\} .
\end{aligned}
$$

Since $A_{1}(t, x) \in \mathcal{F}_{t}$ and $\mathbb{P}_{\delta_{x}}\left(R(t, f) \mid \mathcal{F}_{t}\right)=0$, we have by (3.47) that

$$
M_{1}(t, x)=\frac{1}{t+1} \mathbb{P}_{\delta_{x}}\left(|R(t, f)|^{2} ; A_{1}(t, x)\right)+\frac{t}{t+1} F(t, x),
$$

and

$$
M_{2}(t, x) \leq \frac{2}{t+1} \mathbb{P}_{\delta_{x}}\left(|R(t, f)|^{2} ; A_{2}(t, x)\right)+\frac{2 t}{t+1} \mathbb{P}_{\delta_{x}}\left(\left|S_{t} f(x)\right|^{2} ; A_{2}(t, x)\right) .
$$

Thus we have

$$
F(t+1, x) \leq \frac{t}{t+1} F(t, x)+\frac{1}{t+1}\left(F_{1}(t, x)+F_{2}(t, x)\right),
$$

where

$$
\begin{aligned}
& F_{1}(t, x)=2 \mathbb{P}_{\delta_{x}}\left(|R(t, f)|^{2} ; A_{1}(t, x) \cup A_{2}(t, x)\right), \\
& F_{2}(t, x)=2 t \mathbb{P}_{\delta_{x}}\left(\left|S_{t} f(x)\right|^{2} ; A_{2}(t, x)\right) .
\end{aligned}
$$

Iterating (3.48), we get

$$
\begin{aligned}
F(t+1, x) & \leq \frac{1}{t+1} \sum_{m=0}^{[t]} F_{1}\left(m+\epsilon_{t}, x\right)+\frac{1}{t+1} \sum_{m=0}^{[t]} F_{2}\left(m+\epsilon_{t}, x\right)+\frac{\epsilon_{t}}{t+1} F\left(\epsilon_{t}, x\right) \\
& :=L_{1}(t, x)+L_{2}(t, x)+\frac{\epsilon_{t}}{t+1} F\left(\epsilon_{t}, x\right) .
\end{aligned}
$$

First, we consider $L_{1}(t, x)$. By (2.36) and (2.32), there exist $C>0$ and $r \in \mathbb{N}$ such that, for any $s>0$ and $x \in \mathbb{R}^{d}$

$$
F_{1}(s, x) \leq 2 \mathbb{P}_{\delta_{x}}\left(|R(s, f)|^{2}\right)=2 e^{-\alpha} T_{s}\left(\operatorname{Var}_{\delta .}\left\langle f, X_{1}\right\rangle\right)(x) \leq C\left(1+\|x\|^{r}\right) .
$$

We claim that for any $x \in \mathbb{R}^{d}$,

$$
F_{1}(t, x) \rightarrow 0, \quad \text { as } t \rightarrow \infty .
$$

Then, for any $\epsilon>0$, there exists $K \in \mathbb{N}$ such that $s \geq K$ implies $F_{1}(s, x)<\epsilon$. So, by (3.50), we get that for any $x \in \mathbb{R}^{d}$ and $t>0$,

$$
L_{1}(t, x)=\frac{1}{t+1} \sum_{m=0}^{K-1} F_{1}\left(m+\epsilon_{t}, x\right)+\frac{1}{t+1} \sum_{m=K}^{[t]} F_{1}\left(m+\epsilon_{t}, x\right) \leq \frac{C K}{t+1}\left(1+\|x\|^{r}\right)+\epsilon .
$$


Thus $\lim \sup _{t \rightarrow \infty} L_{1}(t, x) \leq \epsilon$ for any $x$, which implies

$$
\lim _{t \rightarrow \infty} L_{1}(t, x)=0, \quad x \in \mathbb{R}^{d} .
$$

Now we prove the claim (3.51). First, we will show that, for any $x \in \mathbb{R}^{d}$, as $t \rightarrow \infty$,

$$
\mathbb{P}_{\delta_{x}}\left(A_{1}(t, x) \cup A_{2}(t, x)\right) \rightarrow 0 .
$$

By Chebyshev's inequality and (2.38), we have that, for any $x \in \mathbb{R}^{d}$, as $t \rightarrow \infty$,

$$
\mathbb{P}_{\delta_{x}}\left(A_{1}(t, x)\right) \leq c^{-2} e^{-2 \delta t} \mathbb{P}_{\delta_{x}}\left|S_{t} f(x)\right|^{2} \rightarrow 0 .
$$

It is easy to see that, under $\mathbb{P}_{\delta_{x}}$, for any $t>0$,

$$
A_{2}(t, x) \subset\left\{|R(t, f)|>c e^{\delta t}\left(e^{\delta} \sqrt{t+1}-\sqrt{t}\right)\right\} .
$$

Similarly, by Chebyshev's inequality, we have that

$$
\mathbb{P}_{\delta_{x}}\left(A_{2}(t, x)\right) \leq c^{-2} e^{-2 \delta t}\left(e^{\delta} \sqrt{t+1}-\sqrt{t}\right)^{-2} \mathbb{P}_{\delta_{x}}|R(t, f)|^{2}
$$

By (2.24), we get that

$$
\mathbb{P}_{\delta_{x}}|R(t, f)|^{2}=e^{-\alpha} T_{t}\left(\operatorname{Var}_{\delta}\left\langle f, X_{1}\right\rangle\right)(x) \rightarrow e^{-\alpha}\left\langle\operatorname{Var}_{\delta}\left\langle f, X_{1}\right\rangle, \varphi\right\rangle, \quad \text { as } \rightarrow \infty,
$$

which implies $\mathbb{P}_{\delta_{x}}\left(A_{2}(t, x)\right) \rightarrow 0$ for any $x \in \mathbb{R}^{d}$.

In the rest of the proof of (3.51), we will replace $\left(X_{t}, \mathbb{P}_{\mu}\right)$ by $\left(\Lambda_{t}, \mathbf{P}_{\mu}\right)$. Using (3.9) with $s=1$, we have

$$
\begin{aligned}
R(t, f)= & e^{-(\alpha / 2)(t+1)}\left(\left\langle f, \Lambda_{t+1}\right\rangle-\left\langle T_{1}^{\alpha} f, \Lambda_{t}\right\rangle\right) \\
= & e^{-(\alpha / 2)(t+1)}\left(\left\langle f, \tilde{X}_{1}^{t}\right\rangle-\mathbf{P}_{\delta_{x}}\left(\left\langle f, \tilde{X}_{1}^{t}\right\rangle \mid \mathcal{G}_{t}\right)+e^{-(\alpha / 2) t} \sum_{u \in \mathcal{L}_{t}}\left(Y_{1}^{u, t}-y_{1}^{u, t}\right)\right. \\
& +e^{-(\alpha / 2)(t+1)}\left(e^{\alpha}-e^{-\alpha^{*}}\right) e^{-\alpha / 2}\left(\frac{1}{\lambda^{*}}\left\langle f, Z_{t}\right\rangle-\left\langle f, \Lambda_{t}\right\rangle\right) \\
=: & J_{0}(t)+J_{1}(t)+J_{2}(t),
\end{aligned}
$$

where $Y_{1}^{u, t}, y_{1}^{u, t}$ are defined in (3.8). So for any $\epsilon>0$,

$$
\begin{aligned}
F_{1}(t, x) \leq & 6 \mathbf{P}_{\delta_{x}}\left(\left|J_{0}(t)\right|^{2} ; A_{1}(t, x) \cup A_{2}(t, x)\right)+6 \mathbf{P}_{\delta_{x}}\left(\left|J_{1}(t)\right|^{2} ; A_{1}(t, x) \cup A_{2}(t, x)\right) \\
& +6 \mathbf{P}_{\delta_{x}}\left(\left|J_{2}(t)\right|^{2} ; A_{1}(t, x) \cup A_{2}(t, x)\right) \\
=: & F_{11}(t, x)+F_{12}(t, x)+F_{13}(t, x) .
\end{aligned}
$$

For $F_{11}(t, x)$ and $F_{13}(t, x)$, we claim that

$$
\underset{t \rightarrow \infty}{\limsup } \mathbf{P}_{\delta_{x}}\left|J_{0}(t)\right|^{4}<\infty \quad \text { and } \quad \limsup _{t \rightarrow \infty} \mathbf{P}_{\delta_{x}}\left|J_{2}(t)\right|^{4}<\infty .
$$

Then by (3.53),

$$
\begin{aligned}
\limsup _{t \rightarrow \infty} F_{11}(t, x) & \leq 6 \limsup _{t \rightarrow \infty} \mathbf{P}_{\delta_{x}}\left(\left|J_{0}(t)\right|^{2} ;\left|J_{0}(t)\right|^{2}>M\right)+6 M \limsup _{t \rightarrow \infty} \mathbf{P}_{\delta_{x}}\left(A_{1}(t, x) \cup A_{2}(t, x)\right) \\
& \leq 6 M^{-2} \limsup _{t \rightarrow \infty} \mathbf{P}_{\delta_{x}}\left(\left|J_{0}(t)\right|^{4}\right) .
\end{aligned}
$$


Letting $M \rightarrow \infty$, we get $F_{11}(t, x) \rightarrow 0$ as $t \rightarrow \infty$. Similarly, we have $F_{13}(t, x) \rightarrow 0$ as $t \rightarrow \infty$. Now we prove the claim (3.57).

For $J_{0}(t)$, by (2.61), there exists $r \in \mathbb{N}$ such that

$$
\begin{aligned}
\mathbf{P}_{\delta_{x}}\left|J_{0}(t)\right|^{4} & \lesssim e^{-2 \alpha(t+1)} \mathbf{P}_{\delta_{x}}\left(\left\langle 1+\|\cdot\|^{4 r}, \Lambda_{t}\right\rangle+\left\langle 1+\|\cdot\|^{2 r}, \Lambda_{t}\right\rangle^{2}\right) \\
& =e^{-\alpha(t+2)} T_{t}\left(1+\|\cdot\|^{4 r}\right)(x)+e^{-2 \alpha(t+1)} \mathbf{P}_{\delta_{x}}\left\langle 1+\|\cdot\|^{2 r}, \Lambda_{t}\right\rangle^{2} .
\end{aligned}
$$

Thus, by (2.24) and (2.39), we get

$$
\limsup _{t \rightarrow \infty} \mathbf{P}_{\delta_{x}}\left|J_{0}(t)\right|^{4}<\infty .
$$

For $J_{2}(t)$, since, given $\Lambda_{t}, Z_{t}$ is a Poisson random measure with intensity $\lambda^{*} \Lambda_{t}$, we have

$$
\begin{aligned}
e^{-2 \alpha t} \mathbf{P}_{\delta_{x}}\left(\left\langle f, Z_{t}\right\rangle-\lambda^{*}\left\langle f, \Lambda_{t}\right\rangle\right)^{4} & =\lambda^{*} e^{-2 \alpha t} \mathbf{P}_{\delta_{x}}\left\langle f^{4}, \Lambda_{t}\right\rangle+3\left(\lambda^{*}\right)^{2} e^{-2 \alpha t} \mathbf{P}_{\delta_{x}}\left\langle f^{2}, \Lambda_{t}\right\rangle^{2} \\
& =\lambda^{*} e^{-\alpha t} T_{t}\left(f^{4}\right)(x)+3\left(\lambda^{*}\right)^{2} e^{-2 \alpha t} \mathbf{P}_{\delta_{x}}\left\langle f^{2}, \Lambda_{t}\right\rangle^{2} .
\end{aligned}
$$

Thus, by (2.24) and (2.39), we get

$$
\limsup _{t \rightarrow \infty} \mathbf{P}_{\delta_{x}}\left|J_{2}(t)\right|^{4}<\infty
$$

Next, we consider $F_{12}(t, x)$. From the proof of (3.17), we see that (3.17) is also true when $\alpha=2 \gamma(f) b$. So we have $J_{1}(t) \stackrel{d}{\rightarrow} \sqrt{W_{\infty}} G$ where $G$ is a Gaussian random variable. We also have $\mathbf{P}_{\delta_{x}}\left|J_{1}(t)\right|^{2} \rightarrow \mathbf{P}_{\delta_{x}}\left(W_{\infty} G^{2}\right)$.

Let $\Psi_{M}(r)=r$ on $[0, M-1], \Psi_{M}(r)=0$ on $[M, \infty]$, and let $\Psi_{M}$ be linear on $[M-1, M]$. Therefore by (3.53) and (3.55), we have that for any $x \in E$,

$$
\begin{aligned}
\limsup _{t \rightarrow \infty} F_{12}(t, x) & \leq \limsup _{t \rightarrow \infty} 6 \mathbf{P}_{\delta_{x}}\left(\left|J_{1}(t)\right|^{2} ;\left|J_{1}(t)\right|^{2}>M\right)+6 M \limsup _{t \rightarrow \infty} \mathbf{P}_{\delta_{x}}\left(A_{1}(t, x) \cup A_{2}(t, x)\right) \\
& \leq 6 \limsup _{t \rightarrow \infty}\left(\mathbf{P}_{\delta_{x}}\left(\left|J_{1}(t)\right|^{2}\right)-\mathbf{P}_{\delta_{x}}\left(\Psi_{M}\left(\left|J_{1}(t)\right|^{2}\right)\right)\right) \\
& =6\left(\mathbf{P}_{\delta_{x}}\left(W_{\infty} G^{2}\right)-\mathbf{P}_{\delta_{x}}\left(\Psi_{M}\left(W_{\infty} G^{2}\right)\right)\right) .
\end{aligned}
$$

By the monotone convergence theorem, we have that,

$$
\lim _{M \rightarrow \infty} \mathbf{P}_{\delta_{x}}\left(\Psi_{M}\left(W_{\infty} G^{2}\right)\right)=\mathbf{P}_{\delta_{x}}\left(W_{\infty} G^{2}\right),
$$

which implies that $F_{12}(t, x) \rightarrow 0$, as $t \rightarrow \infty$. Therefore, (3.51) is valid.

Now we consider $L_{2}(t, x)$.

$$
\begin{aligned}
F_{2}(t, x) & =2 t \mathbb{P}_{\delta_{x}}\left(\left|S_{t} f(x)\right|^{2} ; A_{2}(t, x)\right) \\
& \leq 2 t c e^{\delta t} \mathbb{P}_{\delta_{x}}\left(\left|S_{t} f(x)\right| ;|R(t, f)|>c e^{\delta t}\left(e^{\delta} \sqrt{t+1}-\sqrt{t}\right)\right) \\
& \leq 2 c^{-1} t e^{-\delta t}\left(e^{\delta} \sqrt{t+1}-\sqrt{t}\right)^{-2} \mathbb{P}_{\delta_{x}}\left(\left|S_{t} f(x)\right| \cdot|R(t, f)|^{2}\right) \\
& \lesssim e^{-\delta t} e^{-\alpha(t+1)} \mathbb{P}_{\delta_{x}}\left(\left|S_{t} f(x)\right|\left\langle\operatorname{Var}_{\delta}\left\langle f, X_{1}\right\rangle, X_{t}\right\rangle\right) \\
& \lesssim e^{-\delta t} \sqrt{\mathbb{P}_{\delta_{x}}\left|S_{t} f(x)\right|^{2}} \sqrt{e^{-2 \alpha t} \mathbb{P}_{\delta_{x}}\left(\left\langle\operatorname{Var}_{\delta}\left\langle f, X_{1}\right\rangle, X_{t}\right\rangle^{2}\right)} .
\end{aligned}
$$


By (2.38) and (2.39), we get $F_{2}(t, x) \rightarrow 0$ as $t \rightarrow \infty$. Thus, for any $\epsilon>0$, there exists $K \in \mathbb{N}$ such that $s \geq K$ implies $F_{2}(s, x)<\epsilon$. It is easy to see that,

$$
\sup _{s \leq K} F_{2}(s, x) \leq \sup _{s \leq K} 2 c^{2} s e^{2 \delta s} \leq 2 c^{2} K e^{2 \delta K} .
$$

Thus, we get

$$
L_{2}(t, x)=\frac{1}{t+1} \sum_{m=0}^{K-1} F_{2}\left(m+\epsilon_{t}, x\right)+\frac{1}{t+1} \sum_{m=K}^{[t]} F_{2}\left(m+\epsilon_{t}, x\right) \leq \frac{2 c^{2} K^{2} e^{2 \delta K}}{t+1}+\epsilon .
$$

Therefore $\lim \sup _{t \rightarrow \infty} L_{2}(t, x) \leq \epsilon$, which implies that $\lim _{t \rightarrow \infty} L_{2}(t, x)=0$.

To finish the proof, we need to show that,

$$
\lim _{t \rightarrow \infty} \frac{\epsilon_{t}}{t+1} F\left(\epsilon_{t}, x\right)=0
$$

By (2.36), we get that for any $x \in \mathbb{R}^{d}$,

$$
\sup _{t>0}\left(\epsilon_{t}\right) F\left(\epsilon_{t}, x\right) \leq \sup _{s \geq 0} \mathbb{P}_{\delta_{x}}\left(S_{s} f(x)\right)^{2}<\infty,
$$

which implies (3.58).

In the following lemma we give a result similar to Lemma 3.2 for the process $I$.

Lemma 3.3 Assume $f \in \mathcal{P}$ satisfies $\alpha=2 \gamma(f) b$. Define

$$
Y_{t}^{*}(f):=t^{-1 / 2} e^{-(\alpha / 2) t}\left(\left\langle f, I_{t}\right\rangle-\mathbb{Q}_{\delta_{x}}\left\langle f, I_{t}\right\rangle\right) .
$$

Then for any $c>0$ and $\delta>0$, we have

$$
\lim _{t \rightarrow \infty} \mathbb{Q}_{\delta_{x}}\left(\left|Y_{t}^{*}(f)\right|^{2} ;\left|Y_{t}^{*}(f)\right|>c e^{\delta t}\right)=0 .
$$

Proof Recall the decomposition in (2.4). Define

$$
\begin{aligned}
S_{t}^{*} & =t^{-1 / 2} e^{-(\alpha / 2) t}\left(\left\langle f, \tilde{X}_{t}\right\rangle-\mathbf{P}_{\delta_{x}}\left\langle f, \widetilde{X}_{t}\right\rangle\right), \\
S_{t} & =t^{-1 / 2} e^{-(\alpha / 2) t}\left(\left\langle f, \Lambda_{t}\right\rangle-\mathbf{P}_{\delta_{x}}\left\langle f, \Lambda_{t}\right\rangle\right),
\end{aligned}
$$

and

$$
\tilde{Y}_{t}=t^{-1 / 2} e^{-(\alpha / 2) t}\left(\left\langle f, I_{t}\right\rangle-\mathbf{P}_{\delta_{x}}\left\langle f, I_{t}\right\rangle\right) .
$$

Then we have $\tilde{Y}_{t}=S_{t}-S_{t}^{*}$. Thus

$$
\begin{aligned}
\mathbf{P}_{\delta_{x}}\left(\left|\tilde{Y}_{t}\right|^{2} ;\left|\tilde{Y}_{t}\right|>c e^{\delta t}\right) \leq & \mathbf{P}_{\delta_{x}}\left(\left|\tilde{Y}_{t}\right|^{2} ;\left|S_{t}\right|>(c / 2) e^{\delta t}\right)+\mathbf{P}_{\delta_{x}}\left(\left|\widetilde{Y}_{t}\right|^{2} ;\left|S_{t}^{*}\right|>(c / 2) e^{\delta t}\right) \\
\leq & 2 \mathbf{P}_{\delta_{x}}\left(\left|S_{t}\right|^{2} ;\left|S_{t}\right|>(c / 2) e^{\delta t}\right)+2 \mathbf{P}_{\delta_{x}}\left(\left|S_{t}^{*}\right|^{2}\right) \\
& +\mathbf{P}_{\delta_{x}}\left(\left|\widetilde{Y}_{t}\right|^{2} ;\left|S_{t}^{*}\right|>(c / 2) e^{\delta t}\right) \\
= & I_{1}(t)+I_{2}(t)+I_{3}(t) .
\end{aligned}
$$


By Lemma 3.2, we have $\lim _{t \rightarrow \infty} I_{1}(t)=0$. By (2.59), we have

$$
I_{2}(t)=2 t^{-1} e^{-\alpha t} \mathbf{V} a r_{\delta_{x}}\left\langle f, \tilde{X}_{t}\right\rangle \rightarrow 0, \quad t \rightarrow \infty .
$$

Since $I_{t}$ and $\widetilde{X}$ are independent, we have

$$
I_{3}(t)=\mathbf{P}_{\delta_{x}}\left(\left|\widetilde{Y}_{t}\right|^{2}\right) \mathbf{P}_{\delta_{x}}\left(\left|S_{t}^{*}\right|>(c / 2) e^{\delta t}\right) .
$$

Since $S_{t}=S_{t}^{*}+\widetilde{Y}_{t}$, and $S_{t}^{*}$ and $\widetilde{Y}_{t}$ are independent, by (2.38), we get

$$
\mathbf{P}_{\delta_{x}}\left(\left|\tilde{Y}_{t}\right|^{2}\right)=\mathbf{P}_{\delta_{x}}\left(\left|S_{t}\right|^{2}\right)-\mathbf{P}_{\delta_{x}}\left(\left.S_{t}^{*}\right|^{2}\right) \rightarrow \rho_{f}^{2}, \quad t \rightarrow \infty .
$$

By Chebyshev's inequality, we have

$$
\mathbf{P}_{\delta_{x}}\left(\left|S_{t}^{*}\right|>(c / 2) e^{\delta t}\right) \leq(c / 2)^{-2} e^{-2 \delta t} \mathbf{P}_{\delta_{x}}\left(\left|S_{t}^{*}\right|^{2}\right) \rightarrow 0, \quad t \rightarrow \infty .
$$

Hence $\lim _{t \rightarrow \infty} I_{3}(t)=0$. Thus

$$
\mathbf{P}_{\delta_{x}}\left(\left|\widetilde{Y}_{t}\right|^{2} ;\left|\widetilde{Y}_{t}\right|>c e^{\delta t}\right) \rightarrow 0 .
$$

Recall that under $\mathbf{P}_{\delta_{x}}, I_{t}=\sum_{j=1}^{N} I_{t}^{j}$, where $I^{j}, j=1, \ldots$ are independent copies of $I$ under $\mathbb{Q}_{\delta_{x}}$, and are independent of $N$. Thus,

$$
\begin{aligned}
\mathbf{P}_{\delta_{x}}\left(\left|\widetilde{Y}_{t}\right|^{2} ;\left|\widetilde{Y}_{t}\right|>c e^{\delta t}\right) & \geq \mathbf{P}_{\delta_{x}}\left(\left|\widetilde{Y}_{t}\right|^{2} ;\left|\widetilde{Y}_{t}\right|>c e^{\delta t}, N=1\right) \\
& =\mathbf{P}_{\delta_{x}}(N=1) \mathbb{Q}_{\delta_{x}}\left(\left|Y_{t}^{*}(f)\right|^{2} ;\left|Y_{t}^{*}(f)\right|>c e^{\delta t}\right) .
\end{aligned}
$$

Since $\mathbf{P}_{\delta_{x}}(N=1)>0$, (3.59) follows easily from (3.60).

Now, we are ready to prove Theorem 1.6.

Proof of Theorem 1.6 The proof is similar to that of Theorem 1.4. Since $\alpha=2 \gamma(f) b$, $f(x)=f_{(c)}(x)+f_{(l)}(x)$. Using Theorem 1.4 for $f_{(l)}$, we have as $t \rightarrow \infty$,

$$
t^{-1 / 2} e^{-(\alpha / 2) t}\left\langle f_{(l)}, \Lambda_{t}\right\rangle \stackrel{d}{\rightarrow} 0
$$

So we only need to prove Theorem 1.6 for the case $f(x)=\sum_{|p|=\gamma(f)} a_{p} \phi_{p}(x)$. We define an $\mathbb{R}^{2}$-valued random variable by

$$
U_{1}(t):=\left(e^{-\alpha t}\left\|\Lambda_{t}\right\|, t^{-1 / 2} e^{-(\alpha / 2) t}\left\langle f, \Lambda_{t}\right\rangle\right)
$$

We need to show that as $t \rightarrow \infty$,

$$
U_{1}(t) \stackrel{d}{\rightarrow}\left(W_{\infty}, \sqrt{W_{\infty}} G_{2}(f)\right),
$$

where $G_{2}(f) \sim \mathcal{N}\left(0, \rho_{f}^{2}\right)$. Let $n>0$ and write

$$
U_{1}(n t)=\left(e^{-\alpha n t}\left\|\Lambda_{n t}\right\|,(n t)^{-1 / 2} e^{-(\alpha / 2) n t}\left\langle f, \Lambda_{n t}\right\rangle\right) .
$$

Recall the representation (3.1). Define

$$
Y_{t}^{u, n}:=((n-1) t)^{-1 / 2} e^{-\alpha(n-1) t / 2}\left\langle f, I_{(n-1) t}^{u, t}\right\rangle \quad \text { and } \quad y_{t}^{u, n}:=\mathbf{P}_{\mu}\left(Y_{t}^{u, n} \mid \mathcal{G}_{t}\right)
$$


$Y_{t}^{u, n}$ has the same distribution as $Y_{t}^{n}:=((n-1) t)^{-1 / 2} e^{-\alpha(n-1) t / 2}\left\langle f, I_{(n-1) t}\right\rangle$ under $\mathbb{Q}_{\delta_{z_{u}(t)}}$. Thus

$$
\begin{aligned}
& (n t)^{-1 / 2} e^{-(\alpha / 2) n t}\left\langle f, \Lambda_{n t}\right\rangle \\
& =(n t)^{-1 / 2} e^{-(\alpha / 2) n t}\left\langle f, \widetilde{X}_{(n-1) t}^{t}\right\rangle+\sqrt{\frac{n-1}{n}} e^{-(\alpha / 2) t} \sum_{u \in \mathcal{L}_{t}} Y_{t}^{u, n} \\
& =(n t)^{-1 / 2} e^{-(\alpha / 2) n t}\left(\left\langle f, \widetilde{X}_{(n-1) t}^{t}\right\rangle-\mathbf{P}_{\mu}\left(\left\langle f, \widetilde{X}_{(n-1) t}^{t}\right\rangle \mid \mathcal{G}_{t}\right)\right)+\sqrt{\frac{n-1}{n}} e^{-(\alpha / 2) t} \sum_{u \in \mathcal{L}_{t}}\left(Y_{t}^{u, n}-y_{t}^{u, n}\right) \\
& \quad+(n t)^{-1 / 2} e^{-(\alpha / 2) n t}\left(e^{-\alpha^{*}(n-1) t}\left\langle T_{(n-1) t} f, \Lambda_{t}\right\rangle+\frac{1}{\lambda^{*}}\left(e^{\alpha(n-1) t}-e^{-\alpha^{*}(n-1) t}\right)\left\langle T_{(n-1) t} f, Z_{t}\right\rangle\right) \\
& =: \\
& : J_{0}^{n}(t)+J_{1}^{n}(t)+J_{2}^{n}(t) .
\end{aligned}
$$

Put $\widetilde{V}_{s}(x):=\mathbf{V} a r_{\delta_{x}}\left\langle f, \widetilde{X}_{s}\right\rangle$. Then by (2.59), there exists $r \in \mathbb{N}$ such that $\widetilde{V}_{s}(x) \lesssim e^{-\alpha^{*} s}(1+$ $\left.\|x\|^{2 r}\right)$. From the definition of $\tilde{X}_{s}^{t}$, we have

$$
\begin{aligned}
\mathbf{P}_{\mu} J_{0}^{n}(t)^{2} & =(n t)^{-1} e^{-\alpha(n t)} \mathbf{P}_{\mu}\left(\left\langle\widetilde{V}_{(n-1) t}, \Lambda_{t}\right\rangle\right) \\
& =(n t)^{-1} e^{-\alpha(n-1) t}\left\langle T_{t}\left(\widetilde{V}_{(n-1) t}, \mu\right\rangle\right. \\
& \lesssim(n t)^{-1} e^{-\alpha(n-1) t} e^{-\alpha^{*}(n-1) t} \rightarrow 0, \quad \text { as } t \rightarrow \infty
\end{aligned}
$$

Since $T_{u} f(x)=e^{-\alpha u / 2} f(x)$,

$$
\begin{aligned}
\left(J_{2}^{n}(t)\right)^{2} & =(n t)^{-1} e^{-(2 n-1) \alpha t}\left(e^{-\alpha^{*}(n-1) t}\left\langle f, \Lambda_{t}\right\rangle+\frac{1}{\lambda^{*}}\left(e^{\alpha(n-1) t}-e^{-\alpha^{*}(n-1) t}\right)\left\langle f, Z_{t}\right\rangle\right)^{2} \\
& \lesssim(n t)^{-1} e^{-(2 n-1) \alpha t}\left\langle f, \Lambda_{t}\right\rangle^{2}+(n t)^{-1} e^{-\alpha t}\left\langle f, Z_{t}\right\rangle^{2}
\end{aligned}
$$

By (2.36), we have

$$
\mathbf{P}_{\mu}\left\langle f, \Lambda_{t}\right\rangle^{2}=\left\langle\mathbf{V} a r_{\delta .}\left\langle f, \Lambda_{t}\right\rangle, \mu\right\rangle+e^{\alpha t}\langle f, \mu\rangle^{2} \lesssim(t+1) e^{\alpha t}
$$

Using an argument similar to that in the proof of (3.14), we can get

$$
\begin{aligned}
e^{-\alpha t} \mathbf{P}_{\mu}\left\langle f, Z_{t}\right\rangle^{2} & =\lambda^{*} e^{-\alpha t} \mathbf{P}_{\mu}\left\langle f^{2}, \Lambda_{t}\right\rangle+\left(\lambda^{*}\right)^{2} e^{-\alpha t} \mathbf{P}_{\mu}\left\langle f, \Lambda_{t}\right\rangle^{2} \\
& =\lambda^{*}\left\langle T_{t}\left(f^{2}\right), \mu\right\rangle+\left(\lambda^{*}\right)^{2} e^{-\alpha t} \mathbf{P}_{\mu}\left\langle f, \Lambda_{t}\right\rangle^{2} \\
& \lesssim \lambda^{*}\left\langle T_{t}\left(f^{2}\right), \mu\right\rangle+(t+1)
\end{aligned}
$$

Thus,

$$
\limsup _{t \rightarrow \infty} \mathbf{P}_{\mu}\left(J_{2}^{n}(t)\right)^{2} \lesssim n^{-1}
$$

Combining (3.63) and (3.65), there exists $c>0$ such that

$$
\limsup _{t \rightarrow \infty} \mathbf{P}_{\mu}\left(J_{0}^{n}(t)+J_{2}^{n}(t)\right)^{2} \leq c / n .
$$


Now we consider $J_{1}^{n}(t)$. We define an $\mathbb{R}^{2}$-valued random variable $U_{2}(n, t)$ by

$$
U_{2}(n, t):=\left(e^{-\alpha t}\left\|\Lambda_{t}\right\|, e^{-(\alpha / 2) t} \sum_{u \in \mathcal{L}_{t}}\left(Y_{t}^{u, n}-y_{t}^{u, n}\right)\right) .
$$

We claim that

$$
U_{2}(n, t) \stackrel{d}{\rightarrow}\left(W_{\infty}, \sqrt{W_{\infty}} G_{2}(f)\right), \quad \text { as } t \rightarrow \infty .
$$

Denote the characteristic function of $U_{2}(n, t)$ under $\mathbf{P}_{\mu}$ by $\kappa_{2}\left(\theta_{1}, \theta_{2}, n, t\right)$. Using an argument similar to that leading to (3.18), we get

$$
\kappa_{2}\left(\theta_{1}, \theta_{2}, n, t\right)=\mathbf{P}_{\mu}\left(\exp \left\{i \theta_{1} e^{-\alpha t}\left\|\Lambda_{t}\right\|\right\} \exp \left\{\lambda^{*}\left\langle h_{t}^{n}\left(\cdot, e^{-(\alpha / 2) t} \theta_{2}\right)-1, \Lambda_{t}\right\rangle\right\}\right),
$$

where $h_{t}^{n}(x, \theta)=\mathbb{Q}_{\delta_{x}} e^{i \theta\left(Y_{t}^{n}-\mathbb{Q}_{\delta_{x}} Y_{t}^{n}\right)}$. Define

$$
e_{t}^{n}(x, \theta):=h_{t}^{n}(x, \theta)-1+\frac{1}{2} \theta^{2} \mathbb{V}_{\delta_{x}} Y_{t}^{n}
$$

and $V_{t}^{n}(x):=\mathbb{V}_{\delta_{x}} Y_{t}^{n}$. Then

$$
\begin{aligned}
& \exp \left\{\lambda^{*}\left(h_{t}^{n}\left(\cdot, e^{-(\alpha / 2) t} \theta_{2}\right)-1, \Lambda_{t}\right)\right\} \\
& =\exp \left\{-\frac{1}{2} \lambda^{*} \theta_{2}^{2} e^{-\alpha t}\left\langle V_{t}^{n}, \Lambda_{t}\right)\right\} \exp \left\{\lambda^{*}\left(e_{t}^{n}\left(\cdot, e^{-(\alpha / 2) t} \theta_{2}\right), \Lambda_{t}\right\rangle\right\} \\
& =: J_{1,1}(n, t) J_{1,2}(n, t) .
\end{aligned}
$$

We first consider $J_{1,1}(n, t)$. By (2.37), we have that as $t \rightarrow \infty$,

$$
e^{-\alpha t}\left\langle\left|\lambda^{*} V_{t}^{n}-\rho_{f}^{2}\right|, \Lambda_{t}\right\rangle \lesssim t^{-1} e^{-\alpha t}\left\langle\left(1+\|x\|^{r}\right), \Lambda_{t}\right\rangle \rightarrow 0 \quad \text { in probability. }
$$

It follows that

$$
\lim _{t \rightarrow \infty} e^{-\alpha t}\left\langle\lambda^{*} V_{t}^{n}, \Lambda_{t}\right\rangle=\lim _{t \rightarrow \infty} e^{-\alpha t}\left\langle\rho_{f}^{2}, \Lambda_{t}\right\rangle=\rho_{f}^{2} W_{\infty} \quad \text { in probability, }
$$

which implies that $\lim _{t \rightarrow \infty} J_{1,1}(n, t)=\exp \left\{-\frac{1}{2} \theta_{2}^{2} \rho_{f}^{2} W_{\infty}\right\}$.

For $J_{1,2}(n, t)$, by (3.5), we have, for any $\epsilon>0$,

$$
\begin{aligned}
\left|e_{t}^{n}\left(x, e^{-(\alpha / 2) t} \theta_{2}\right)\right| \leq & \frac{1}{6}\left|\theta_{2}\right|^{3} e^{-\frac{3}{2} \alpha t} \mathbb{Q}_{\delta_{x}}\left(\left|Y_{t}^{n}-\mathbb{Q}_{\delta_{x}} Y_{t}^{n}\right|^{3} ;\left|Y_{t}^{n}-\mathbb{Q}_{\delta_{x}} Y_{t}^{n}\right|<\epsilon e^{\alpha t / 2}\right) \\
& +\theta_{2}^{2} e^{-\alpha t} \mathbb{Q}_{\delta_{x}}\left(\left|Y_{t}^{n}-\mathbb{Q}_{\delta_{x}} Y_{t}^{n}\right|^{2} ;\left|Y_{t}^{n}-\mathbb{Q}_{\delta_{x}} Y_{t}^{n}\right| \geq \epsilon e^{\alpha t / 2}\right) \\
\leq & \frac{\epsilon}{6}|\theta|_{2}^{3} e^{-\alpha t} \mathbb{Q}_{\delta_{x}}\left(\left|Y_{t}^{n}-\mathbb{Q}_{\delta_{x}} Y_{t}^{n}\right|^{2}\right) \\
& +\theta_{2}^{2} e^{-\alpha t} \mathbb{Q}_{\delta_{x}}\left(\left|Y_{t}^{n}-\mathbb{Q}_{\delta_{x}} Y_{t}^{n}\right|^{2} ;\left|Y_{t}^{n}-\mathbb{Q}_{\delta_{x}} Y_{t}^{n}\right| \geq \epsilon e^{\alpha t / 2}\right) \\
= & \frac{\epsilon}{6}|\theta|_{2}^{3} e^{-\alpha t} V_{t}^{n}(x)+\theta_{2}^{2} e^{-\alpha t} F_{t}^{n}(x),
\end{aligned}
$$

where $F_{t}^{n}(x)=\mathbb{Q}_{\delta_{x}}\left(\left|Y_{t}^{n}-\mathbb{Q}_{\delta_{x}} Y_{t}^{n}\right|^{2} ;\left|Y_{t}^{n}-\mathbb{Q}_{\delta_{x}} Y_{t}^{n}\right| \geq \epsilon e^{\alpha t / 2}\right)$. It follows from Lemma 3.3 that $\lim _{t \rightarrow \infty} F_{t}^{n}(x)=0$. By (2.53), we also have

$$
F_{t}^{n}(x) \leq \mathbb{Q}_{\delta_{x}}\left(\left|Y_{t}^{n}-\mathbb{Q}_{\delta_{x}} Y_{t}^{n}\right|^{2}\right) \lesssim 1+\|x\|^{2 r} .
$$


Note that

$$
e^{-\alpha t} \mathbf{P}_{\mu}\left\langle F_{t}^{n}(x), \Lambda_{t}\right\rangle=\left\langle T_{t}\left(F_{t}^{n}\right), \mu\right\rangle .
$$

Thus by the dominated convergence theorem, we get $\lim _{t \rightarrow \infty} e^{-\alpha t} \mathbf{P}_{\mu}\left\langle F_{t}^{n}(x), \Lambda_{t}\right\rangle=0$. It follows that $e^{-\alpha t}\left\langle F_{t}^{n}(x), \Lambda_{t}\right\rangle \rightarrow 0$ in probability. Furthermore from (3.68), we obtain that as $t \rightarrow \infty$,

$$
\frac{\epsilon}{6} \theta_{2}^{3} e^{-\alpha t}\left\langle V_{t}^{n}, \Lambda_{t}\right\rangle \rightarrow \frac{\epsilon}{6 \lambda^{*}} \theta_{2}^{3} \rho_{f}^{2} W_{\infty} \quad \text { in probability. }
$$

Thus, letting $\epsilon \rightarrow 0$, we get that as $t \rightarrow \infty$,

$$
\left\langle\left|e_{t}^{n}\left(x, e^{-(\alpha / 2) t} \theta_{2}\right)\right|, \Lambda_{t}\right\rangle \rightarrow 0 \quad \text { in probability, }
$$

which implies $J_{1,2}(n, t) \rightarrow 1$ in probability, as $t \rightarrow \infty$.

Thus, when $t \rightarrow \infty$,

$$
\exp \left\{\lambda^{*}\left\langle h_{t}^{n}\left(\cdot, e^{-(\alpha / 2) t} \theta_{2}\right)-1, \Lambda_{t}\right\rangle\right\} \rightarrow \exp \left\{-\frac{1}{2} \theta_{2}^{2} \rho_{f}^{2} W_{\infty}\right\}
$$

in probability. Since $h_{t}^{n}(x, \theta)$ is a characteristic function, its real part is less than 1 , which implies

$$
\left|\exp \left\{\lambda^{*}\left\{h_{t}^{n}\left(\cdot, e^{-(\alpha / 2) t} \theta_{2}\right)-1, \Lambda_{t}\right)\right\}\right| \leq 1
$$

So by the dominated convergence theorem, we get that

$$
\lim _{t \rightarrow \infty} \kappa_{2}\left(\theta_{1}, \theta_{2}, n, t\right)=\mathbf{P}_{\mu} \exp \left\{i \theta_{1} W_{\infty}\right\} \exp \left\{-\frac{1}{2} \theta_{2}^{2} \rho_{f}^{2} W_{\infty}\right\},
$$

which implies our claim (3.67). By (3.67) and the fact that $e^{-\alpha n t}\left\|\Lambda_{n t}\right\|-e^{-\alpha t}\left\|\Lambda_{t}\right\| \rightarrow 0$, as $t \rightarrow \infty$ in probability, we easily get

$$
U_{3}(n, t):=\left(e^{-\alpha n t}\left\|\Lambda_{n t}\right\|, J_{1}^{n}(t)\right) \stackrel{d}{\rightarrow}\left(W_{\infty}, \sqrt{\frac{n-1}{n}} \sqrt{W_{\infty}} G_{2}(f)\right) .
$$

Let $\mathcal{D}(n t)$ and $\widetilde{\mathcal{D}}^{n}(t)$ be the distributions of $U_{1}(n t)$ and $U_{3}(n, t)$ respectively, and let $\mathcal{D}^{n}$ and $\mathcal{D}$ be the distributions of $\left(W_{\infty}, \sqrt{\frac{n-1}{n}} \sqrt{W_{\infty}} G_{2}(f)\right)$ and $\left(W_{\infty}, \sqrt{W_{\infty}} G_{2}(f)\right)$ respectively. Then, using (3.4), we have

$$
\begin{aligned}
\limsup _{t \rightarrow \infty} \beta(\mathcal{D}(n t), \mathcal{D}) & \leq \limsup _{t \rightarrow \infty}\left[\beta\left(\mathcal{D}(n t), \widetilde{\mathcal{D}}^{n}(t)\right)+\beta\left(\widetilde{\mathcal{D}}^{n}(t), \mathcal{D}^{n}\right)+\beta\left(\mathcal{D}^{n}, \mathcal{D}\right)\right] \\
& \leq \limsup _{t \rightarrow \infty}\left(\mathbf{P}_{\mu}\left(J_{0}^{n}(t)+J_{2}^{n}(t)\right)^{2}\right)^{1 / 2}+0+\beta\left(\mathcal{D}^{n}, \mathcal{D}\right) .
\end{aligned}
$$

Using this and the definition of $\limsup _{t \rightarrow \infty}$, we easily get that

$$
\limsup _{t \rightarrow \infty} \beta(\mathcal{D}(t), \mathcal{D})=\limsup _{t \rightarrow \infty} \beta(\mathcal{D}(n t), \mathcal{D}) \leq \sqrt{c / n}+\beta\left(\mathcal{D}^{n}, \mathcal{D}\right) .
$$

Letting $n \rightarrow \infty$, we get $\lim \sup _{t \rightarrow \infty} \beta(\mathcal{D}(t), \mathcal{D})=0$. The proof is now complete. 
Proof of Theorem 1.13 First note that

$$
\begin{aligned}
& t^{-1 / 2}\left\|X_{t}\right\|^{-1 / 2}\left(\left\langle f, X_{t}\right\rangle-\sum_{\gamma(f) \leq m<\alpha / 2 b} e^{(\alpha-m b) t} \sum_{|p|=m} a_{p} H_{\infty}^{p}\right) \\
& \quad=t^{-1 / 2}\left\|X_{t}\right\|^{-1 / 2}\left\langle f_{(c l)}, X_{t}\right\rangle+t^{-1 / 2}\left\|X_{t}\right\|^{-1 / 2}\left(\left\langle f_{(s)}, X_{t}\right\rangle-\sum_{n=1}^{k} e^{(\alpha-m b) t} \sum_{|p|=m} a_{p} H_{\infty}^{p}\right) \\
& =: J_{1}(t)+J_{2}(t),
\end{aligned}
$$

where $f_{(c l)}=f_{(l)}+f_{(c)}$. By the definition of $f_{(s)}$, we have $\left(f_{(s)}\right)_{(c)}=0$. Then using Theorem 1.10 for $f_{(s)}$, we have

$$
\left\|X_{t}\right\|^{-1 / 2}\left(\left\langle f_{(s)}, X_{t}\right\rangle-\sum_{n=1}^{k} e^{(\alpha-m b) t} \sum_{|p|=m} a_{p} H_{\infty}^{p}\right) \stackrel{d}{\rightarrow} G_{1}\left(f_{(s)}\right) .
$$

Thus

$$
J_{2}(t) \stackrel{d}{\rightarrow} 0, \quad t \rightarrow \infty .
$$

Since $\alpha=2 \gamma\left(f_{(c l)}\right) b$, so using Theorem 1.6 for $f_{(c l)}$, we have

$$
\left.\left(e^{-\alpha t}\left\|X_{t}\right\|, J_{1}(t)\right)\right) \stackrel{d}{\rightarrow}\left(W^{*}, G_{2}\left(f_{(c l)}\right)\right),
$$

where $G_{2}\left(f_{(c l)}\right) \sim \mathcal{N}\left(0, \rho_{f_{(c l)}}^{2}\right)$. By (1.17), we have $\rho_{f_{(c l)}}^{2}=A \sum_{|p|=\alpha / 2 b}\left(a_{p}\right)^{2}$. Combing (3.74) and (3.75), we arrive at the conclusion of Theorem 1.13.

Acknowledgements We thank Zenghu Li and the two referees for helpful comments on the first version of this paper.

\section{References}

1. Adamczak, R., Miłoś, P.: CLT for Ornstein-Uhlenbeck branching particle system. Preprint (2011). arXiv: 1111.4559

2. Asmussen, S., Hering, H.: Strong limit theorems for general supercritical branching processes with applications to branching diffusions. Z. Wahrs. Verw. Gebiete 36(3), 195-212 (1976)

3. Asmussen, S., Hering, H.: Strong limit theorems for supercritical immigration branching processes. Math. Scand. 39(2), 327-342 (1977)

4. Asmussen, S., Hering, H.: Branching Processes. Birkhäuser, Boston (1983)

5. Asmussen, S., Keiding, N.: Martingale central limit theorems and asymptotic estimation theory for multitype branching processes. Adv. Appl. Probab. 10, 109-129 (1978)

6. Athreya, K.B.: Limit theorems for multitype continuous time Markov branching processes I: The case of an eigenvector linear functional. Z. Wahrs. Verw. Gebiete 12, 320-332 (1969)

7. Athreya, K.B.: Limit theorems for multitype continuous time Markov branching processes II: The case of an arbitrary linear functional. Z. Wahrs. Verw. Gebiete 13, 204-214 (1969)

8. Athreya, K.B.: Some refinements in the theory of supercritical multitype Markov branching processes. Z. Wahrs. Verw. Gebiete 20, 47-57 (1971)

9. Berestycki, J., Kyprianou, A.E., Murillo-Salas, A.: The prolific backbone for supercritical superprocesses. Stoch. Proc. Appl. 121, 1315-1331 (2011)

10. Conner, H.E.: Asymptotic behavior of averaging processes for a branching process of restricted Brownian particles. J. Math. Anal. Appl. 20, 464-479 (1967)

11. Davis, A.W.: Branching-diffusion processes with no absorbing boundaries, I. J. Math. Anal. Appl. 18, 276-296 (1967) 
12. Dudley, R.M.: Real Analysis and Probability. Cambridge University Press, Cambridge (2002)

13. Dynkin, E.B.: Superprocesses and partial differential equations. Ann. Probab. 21, 1185-1262 (1993)

14. Dynkin, E.B., Kuznetsov, S.E.: N-Measure for branching exit Markov system and their applications to differential equations. Probab. Theory Rel. Fields 130, 135-150 (2004)

15. El Karoui, N., Roelly, S.: Propriétés de martingales, explosion et représentation de Lévy-Khintchine d'une classe de processus de branchment à valeurs mesures. Stoch. Proc. Appl. 38, 239-266 (1991)

16. Engländer, J.: Law of large numbers for superdiffusions: the non-ergodic case. Ann. Inst. H. Poincaré Probab. Statist. 45, 1-6 (2009)

17. Engländer, J., Harris, S.C., Kyprianou, A.E.: Strong law of large numbers for branching diffusions. Ann. Inst. H. Poincaré Probab. Statist. 46, 279-298 (2010)

18. Engländer, J., Winter, A.: Law of large numbers for a class of superdiffusions. Ann. Inst. H. Poincaré Probab. Statist. 42, 171-185 (2006)

19. Erdélyi, A., Magnus, W., Oberhettinger, F., Tricomi, F.G.: Higher Transcendental Functions, vol. II. McGraw-Hill, New York (1953)

20. Hardy, R., Harris, S.C.: A spine aproach to branching diffusions with applications to $L^{p}$-convergence of martingales. In: Séminaire de Probabilités XLII. Lecture Notes in Math., vol. 1979, pp. 281-330 (2009)

21. Kesten, H., Stigum, B.P.: A limit theorem for multidimensional Galton-Watson processes. Ann. Math. Statist. 37, 1211-1223 (1966)

22. Kesten, H., Stigum, B.P.: Additional limit theorems for indecomposable multidimensional GaltonWatson processes. Ann. Math. Statist. 37, 1463-1481 (1966)

23. Kyprianou, A.E.: Introductory Lectures on Fluctuations of Levy Processes with Applications. Springer, Berlin (2006)

24. Li, Z.: Skew convolution semigroups and related immigration processes. Theory Probab. Appl. 46, 274296 (2003)

25. Li, Z.: Measure-Valued Branching Markov Processes. Springer, Heidelberg (2011)

26. Liu, R., Ren, Y.-X., Song, R.: $L \log L$ criterion for a class of superdiffusions. J. Appl. Probab. 46, 479496 (2009)

27. Liu, R., Ren, Y.-X., Song, R.: Strong law of large numbers for a class of superdiffusions. Acta Appl. Math. 123, 73-97 (2013)

28. Metafune, G., Pallara, D.: Spectrum of Ornstein-Uhlenbeck operators in $\mathcal{L}^{p}$ spaces with respect to invariant measures. J. Funct. Anal. 196, 40-60 (2002)

29. Miłoś, P.: Spatial CLT for the supercritical Ornstein-Uhlenbeck superprocess. Preprint (2012). arXiv:1203.6661

30. Ren, Y.-X., Song, R., Zhang, R.: Central limit theorems for supercritical branching Markov processes. Preprint (2013). arXiv:1305.0610

31. Watanabe, S.: Limit theorem for a class of branching processes. In: 1967 Markov Processes and Potential Theory, Madison, Wis., 1967. Proc. Sympos. Math. Res. Center, pp. 205-232. Wiley, New York (1967) 